\title{
Solving the grocery backroom layout problem
}

\author{
Maria Pires, Elsa Silva \& Pedro Amorim
}

To cite this article: Maria Pires, Elsa Silva \& Pedro Amorim (2020): Solving the grocery backroom layout problem, International Journal of Production Research, DOI: 10.1080/00207543.2019.1708990

To link to this article: https://doi.org/10.1080/00207543.2019.1708990

曲 Published online: 09 Jan 2020.

ए马

Submit your article to this journal $₫$

Џ Article views: 85

Q View related articles ¿

View Crossmark data ¿

Citing articles: 1 View citing articles $\square$ 


\title{
Solving the grocery backroom layout problem
}

\author{
Maria Pires*, Elsa Silva and Pedro Amorim \\ INESC TEC, Faculdade de Engenharia, Universidade do Porto, Porto, Portugal
}

(Received 4 March 2019; accepted 18 December 2019)

\begin{abstract}
The backroom of retail stores has structural differences when compared with other warehouses and distribution centres, which are more traditionally studied in the literature. This paper presents a mathematical optimisation approach for an unequal area facility layout problem, applied in designing the backroom layout in grocery retail. A set of rectangular facilities (backroom departments) with given area requirements has to be placed, without overlapping, on a limited floor space (backroom area), which can have a regular or an irregular shape. The objective is to find the location and format of the storage departments, such that the walking distances in the store by store employees are minimised. The proposed approach is tested in a European grocery retailer. In the computational experiments, several real store layouts are compared with the ones suggested by the proposed model. The decrease in the walking distances is, on average, 30 percent. In order to understand what the current designers' strategy is, a set of scenarios was created and compared with the real layouts. Each scenario ignores a characteristic of the problem. The goal is to understand what aspect designers are currently discarding. The findings indicate that, currently, designers neglect the different replenishment frequencies of storage departments.
\end{abstract}

Keywords: backroom layout problem; grocery retail; strategic planning; mixed integer programming

\section{Introduction}

Retailers primary deal with finished goods inventory and often stock products in anticipation of customers demand. For that reason, backrooms, which occupy on average 20 percent of retail stores space, have a major importance in retail (Dunne, Lusch, and Carver 1999).

Retail companies may attempt to avoid keeping backroom inventory and favour 'one-touch replenishment' policies, where the products go from the unloading dock directly to the sales area. Nevertheless, there are a number of reasons why retailers continue to keep backroom inventory, such as (i) storing more products per unit of floor space in the backroom compared with the sales floor, (ii) having a backroom inventory that can act as a buffer when deliveries are uncertain or imperfect and when demand forecasts are not accurate, (iii) coping with some bulky or fast moving products where the shelf space available may not be sufficient to unload all products directly into the sales floor, (iv) storing and preparing perishable products, which in the sales area would quickly deteriorate, and (v) offering new opportunities for omnichannel retailing.

Previous studies indicate that backroom operations can have a significant impact on the overall store costs. In-store operations can account for up to 50 percent of total costs in a retailer supply chain and backrooms are responsible for a significant share of these costs (Van Zelst et al. 2009; Kuhn and Sternbeck 2013). Despite this fact, research concerning backrooms has been rare. Researchers have been focused on the optimisation of processes between the Distribution Centres (DCs) and stores as well as on designing the layouts of warehouses and sales area (Drira, Pierreval, and Hajri-Gabouj 2007; Anjos and Vieira 2017). Hence, despite of the importance of layout design in several sectors, most of the previous research has been focusing on manufacturing and distribution. Furthermore, the sales area layout has also been studied due to its direct impact on store sales (Turley and Chebat 2002; Aloysius and Binu 2013; Ozgormus 2015).

In practice, when designing the layout of their stores, retailers understandably pay closer attention to the sales area, i.e. the place that creates direct value to the store. Backrooms, on the other hand, tend to attract less attention and are not as well understood, especially regarding their impact on store performance. Hence, the design of the backroom areas is mainly established empirically, based on the perception of the architect. In order to improve this process, designers should also rely on formal means to assist the design process, rather than follow ad-hoc procedures (Pires et al. 2016).

The scope of this research involves answering the research question of how to effectively design the layout of backroom storage areas. This involves structuring and proposing mathematical models to help retailers adjust backroom layouts

*Corresponding author. Email: maria.pires@fe.up.pt 
to their needs. We propose an effective analytic method for designing backroom layouts considering walking distances, departments adjacency, sales areas layout and other physical constraints. Moreover, the application of this method to design retail backrooms is illustrated with a case study of an European retailer. Due to the lack of literature in this research topic, our problem has relevance and contribution in two existing streams of the literature: facility layout and retail operations.

The remainder of the paper is organised as follows. The next section provides a description of the backroom Layout Problem (BLP). Section 3 reviews the literature concerning the BLP related topics. Section 4 describes the mathematical model used to tackle this problem. Then, the results obtained and their implications are discussed in Section 5. Lastly, Section 6 concludes the paper and provides insights into possible future works.

\section{Problem framing}

The BLP is a strategic decision that consists on positioning the storage departments in the best possible location of the backroom in order to minimise the walking distances in the store, i.e. between the backroom and sales area. An example of a store layout is presented in Figure 1. The BLP is challenging, due to backroom particularities. Backrooms coexist with the sales area, which makes them a unique type of warehouses. In this problem, the walking distances made between storage departments (in the backroom) and the sales area departments/zones depend on the shelf replenishment frequencies. Departments with higher rotation or bulkier products will more likely need to be replenished more often during the day. For that reason, these departments should be nearer to the input/output (I/O) points to the sales area (i.e. backroom doors). However, they should not be located all together in order to avoid congestion.

Space constraints on backrooms depend heavily on the type of store. Larger stores located far from city centres have fewer space constraints, one reason being the cheaper cost per unit area. However, when it comes to convenience stores located in urban centres, and often in buildings, the constraints related with space (available construction space) and architectural aspects (e.g. irregular shapes) are tighter.

Products in grocery stores are generally separated into storage departments, depending on the product segment (category) and storage requirements (temperature and humidity). Products that require different storage temperatures should be placed in different departments, for instance frozen $\left(-18\right.$ to $\left.-35^{\circ} \mathrm{C}\right)$ and chilled $\left(1-4^{\circ} \mathrm{C}\right)$ products. Also, to ensure food safety,

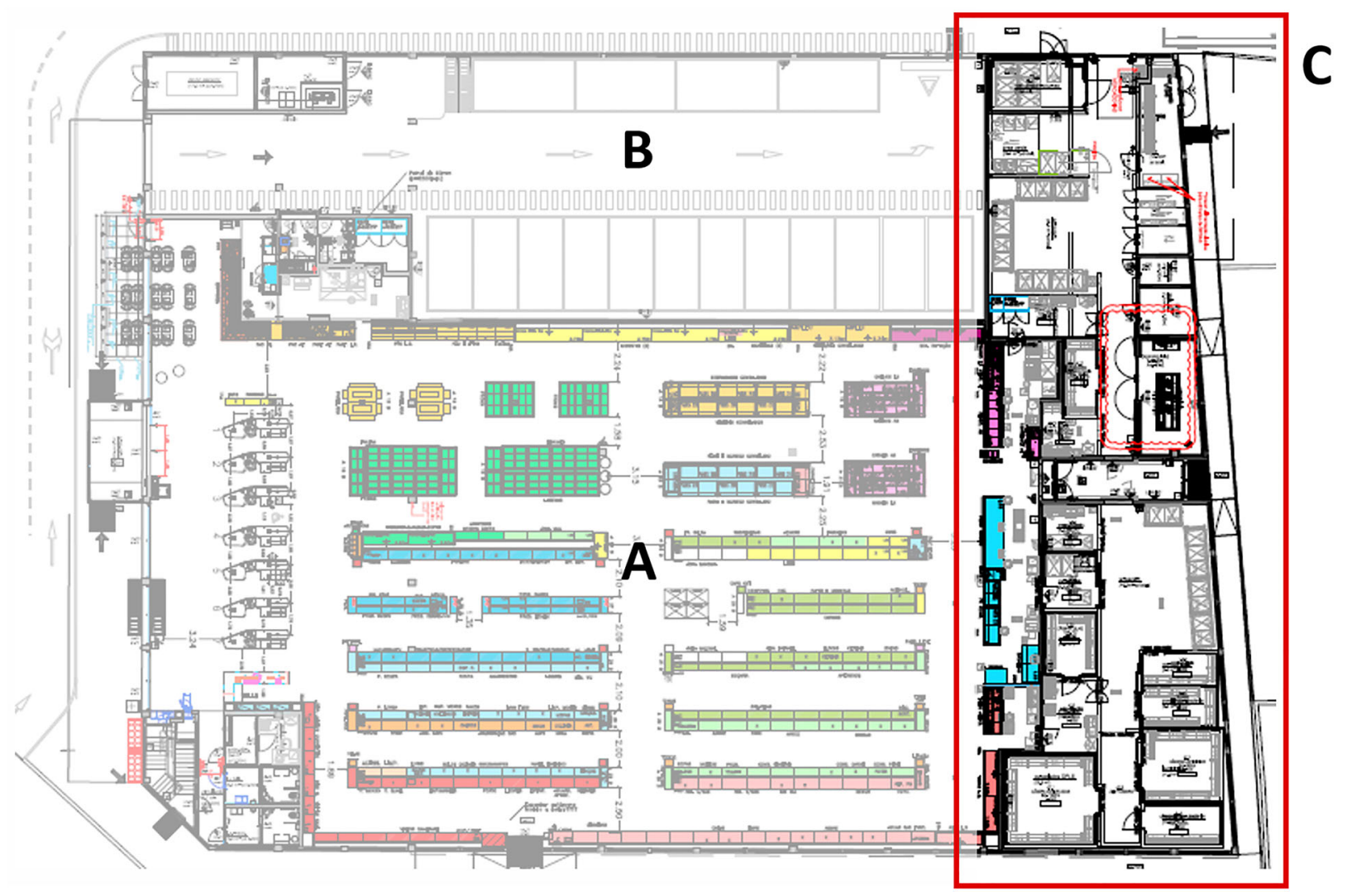

Figure 1. Backroom of an existing store. 'A' represents the sales area, 'B' the unloading dock and ' $\mathrm{C}$ ' is the backroom. 
some products should be separated. For instance, white and red meat should be apart to avoid food contamination. The same happens with raw and cooked food.

In addition to storage departments, service counters and preparation areas are also located in the backroom. Here, products are prepared before going to the sales area or according to customers' requests. These areas should be located near the corresponding departments in the sales area and backroom. When locating departments, it is also necessary to consider energy efficiency aspects. For instance, refrigerated temperature departments (frozen and chilled) should be together, in order to avoid heat loss. Another important decision concerns the aisles design that consume valuable space. In practice, the aisles connect all the storage departments, i.e. all departments should be accessible. Furthermore, since the aisles are not refrigerated, it is common to use this space as storage for the food and nonfood products, which do not require temperature control. Thus, it is required that the area that links all the refrigerated departments is a single undivided polygon with enough width to move the pallet truck and that also has enough space to store the food and nonfood stock.

Lastly, other important particularity is the disposition of the departments. In practice, designers tend to allocate the departments in the perimeter of the backroom in order to allow a larger space in the middle, for ambient products, promotions and buffers.

In a nutshell, the goal of the BLP is to locate the departments in the perimeter of the backroom in order to minimise the distances within the store. In this problem, there are five types of departments: (i) storage departments at ambient temperature (which are located in the middle area of the backroom), (ii) refrigerated storage departments, (iii) frozen storage departments, (iv) preparation departments and, lastly, (v) technical departments. With the exception of storage departments at ambient temperature, all other departments are separate departments. In addition, with the exception of technical departments, all other departments depend on the sales area and, therefore, use the I/O points to the sales area.

\section{Literature review}

The BLP is related to two research streams: retail operations and facility layout. In this section, we will address the existing literature concerning these research topics, while we draw parallels with the BLP.

\subsection{Backroom related topics in retail operations}

Grocery stores are usually arranged into two main areas: sales area and backroom. Whereas the sales area is dedicated to customers, the backroom supports the in-store operations, which are usually complex in a grocery store. Generally, products flow into the store through a receiving area/unloading dock in the backroom before being placed on shelves. Store employees then unload and inspect products arriving from upstream distribution centres and/or suppliers and carry out activities such as stocking and replenishing store shelves. In the sales area, customers view and select products from shelves and exit the store after payment (Fisher 2004; Pires et al. 2015). Items delivered to the retail store by internal distribution centres and/or external suppliers that cannot immediately fit in dedicated retail shelves are typically stocked in a backroom and replenished into the retail shelves when shelf inventory reaches a threshold or has run out (out-of-shelf).

The literature on retail store operations is vast and includes both operational execution and service improvement topics (Mou, Robb, and DeHoratius 2017). Some of the most researched topics in this field include 'Inventory Management' (Chandra and Grabis 2008; Bijvank and Vis 2011), 'Assortment and Display' (Hübner and Kuhn 2012) and 'Shelf Space Allocation' (Bianchi-Aguiar et al. 2018). Store operations also include the physical layout of a retail store, which is closely linked with backroom layout planning. However, the literature concerning grocery stores layout is still limited (Inglay and Dhalla 2010).

The revenue of a grocery store depends on the clients purchases (type and quantity of products), which are highly influenced by the store design (Cil 2012). The impulse purchases, i.e. the unplanned purchases, are the ones that are more influenced by the store layout (Mohan, Sivakumaran, and Sharma 2013). Adjacency plays an important part in the customer's propensity to purchase items related to the ones that he/she had already planned to buy, but that were not in the shopping list. Thus, there is a trade-off between stimulating impulse purchases and increasing a customer's path length, which should be considered while designing a store.

Surjandari and Seruni (2010) unveiled category association rules by analysing market basket data, and then used this information to determine a product placement layout. The sizes of the departments (product category areas) in this paper are fixed in a way that only the locations of products are chosen. Peng (2011) addressed the store layout problem by maximising impulse purchases. The author first defines the must-have items in a grid layout store and then uses an algorithm to distribute these items across the store to increase impulse purchases. The generated layout is improved by using a simulated annealing (SA) metaheuristic. The size of the departments is also assumed to be fixed and equal. Cil (2012) examines the layout strategy of supermarket retail stores by using buying association measures to create a category correlation matrix. The size 
of the departments considered in this work is also fixed. A model and solution approach was developed by Yapicioglu and Smith (2012), using tabu search, for the design of the block layout of a store with a racetrack aisle network. Similarly to Surjandari and Seruni (2010), Aloysius and Binu (2013) presented an approach to product placement in supermarkets that aims to maximise impulse purchases by using market basket data analysis. The authors used data mining techniques to explore frequent patterns in the shopping lists of customers. A mixed integer mathematical model hybridised with both tabu search and genetic algorithm (GA) was proposed by Ozcan and Esnaf (2013) to design a bookstore layout. Pinto, Soares, and Brazdil (2015) combined regression models and a Particle Swarm Optimisation metaheuristic to optimise space allocation with the goal of maximising the predicted sales. More recently, Altuntas and Altuntas (2017) proposed a two-step approach based on utility mining to a derive store layout. Furthermore, the authors conducted a case study in a supermarket to illustrate the approach.

Generally, the objective function in the store layout problem is to maximise the revenue, either by increasing impulse purchases or by the client satisfaction regarding departments and adjacency (Botsali 2007). Also, the sizes of the departments are generally fixed, and sometimes assumed equal sized, so only the locations of products are chosen. In terms of the methods applied, approximated methods are the most used, motivated by the difficulty in solving models to optimality. As a final remark, it is worth noticing that in none of the aforementioned papers the BLP was addressed together with the sales layout.

\subsection{Facility layout}

Facility layout problems (FLPs) are a general class of operations research problems concerned with finding the optimal arrangement of a given number of non-overlapping indivisible departments within a given facility (Anjos and Vieira 2017). This is a challenging combinatorial optimisation problem encountered in many service and manufacturing organisations. The aim is to determine the most effective department arrangement within a facility. In this problem several objectives may be considered, such as minimising the material handling costs or maximising space utilisation. The costs considered are commonly represented by the sum of the products of the weighted rectilinear distance and the material-handling flow between the centroids (Paes, Pessoa, and Vidal 2017).

The FLP is NP-hard in general, so solving it to global optimality in reasonable time is often difficult. Indeed, the restricted version of the problem where the dimensions of the departments are all equal and fixed, and the optimisation is taken over a fixed finite set of possible locations for the departments, is already an NP-hard problem. The constraints of the basic FLP problem encompass the department shape and location requirements. The department shape requirements include the required area and dimensions (height and width). The department location requirements include the non-overlap of departments within the facility.

There are three main classes of FLPs. The row layout problems, Unequal-Areas FLPs, and multi-floor FLPs. Onedimensional facilities lead to row FLPs. The goal of this problem is to assign departments to rows and to position them within the row. The Unequal-Areas FLP is concerned with finding the optimal arrangement of a given number of departments with varying areas so as to minimise the total expected cost of flows inside the facility. Unlike in the row FLPs, the dimensions of each department are optimised (subject to the area requirement) (Armour and Buffa 1963). The multifloor FLP involves finding the optimal arrangement of departments in a facility with multiple floors. The problem tackled in this paper relates to the Unequal-Areas FLPs (UA-FLPs).

Various methods and procedures have been proposed to solve the UA-FLP and can be classified into exact methods, heuristics and meta-heuristics, and math-heuristics. Moreover, most of the proposed approaches are two-stage methodologies, where the first stage determines the relative location of the departments, and the second stage obtains the final layout via a mathematical optimisation model. The main differences between the different approaches are in the first-stage algorithms.

Kim and Kim (2000) developed a MIP model for the layout planning problem with the objective of minimising the sum of rectilinear distances weighted by flow amounts between input and output points of the facilities. Barbosa-Povoa, Mateus, and Novais (2001) proposed a MILP where binary variables are introduced to characterise topological choices (e.g. equipment orientations) and continuous variables to describe the distances and locations involved. Wu and Appleton (2002) presented a method to solve the layout and aisle structure problems simultaneously. The method decomposes the problem into two stages. The first stage minimises the material handling cost with aisle distance, and the second stage optimises the aisle structure. A representation of a slicing floorplan is introduced for the optimisation by genetic algorithms. Lee and Lee (2002) proposed a shape-based block layout approach using a hybrid genetic algorithm in which the objective function minimises the total material handling cost and maximises the space utilisation. Osman, Georgy, and Ibrahim (2003) integrated the graphical capabilities of computer-aided design (CAD) with a genetic algorithm. Later, Wang, $\mathrm{Hu}$, and $\mathrm{Ku}(2005)$ presented a study that combines a GA with analysis of variance (ANOVA). By reformulating the FLP with a sequence-pair representation, Meller, Chen, and Sherali (2007) were able to solve problems with up to eleven facilities. Also, Konak et al. (2006) modelled the facility area constraints exactly using a set of linear constraints derived from 
Table 1. Review of UA-FLP approaches in the literature.

\begin{tabular}{|c|c|c|c|c|c|c|c|}
\hline Author & Var. & Dimens. & Irr.dep. & Irr.fac. & Rotation & O.F. & Sol. \\
\hline Kim and Kim (2000) & $\mathrm{C}$ & - & - & - & $\checkmark$ & DB & $\mathrm{H}$ \\
\hline Barbosa-Povoa, Mateus, and Novais (2001) & $\mathrm{C}$ & $\checkmark$ & - & - & $\checkmark$ & $\mathrm{CB}$ & $\mathrm{E}$ \\
\hline Wu and Appleton (2002) & $\mathrm{C}$ & $\checkmark$ & - & - & - & $\mathrm{DB}$ & $\mathrm{H}$ \\
\hline Lee and Lee (2002) & $\mathrm{D}$ & $\checkmark$ & - & - & - & $\mathrm{DB}+\mathrm{SUB}$ & $\mathrm{H}$ \\
\hline Osman, Georgy, and Ibrahim (2003) & $\mathrm{D}$ & $\checkmark$ & - & $\checkmark$ & - & DB & $\mathrm{H}$ \\
\hline Wang, $\mathrm{Hu}$, and $\mathrm{Ku}(2005)$ & $\mathrm{D}$ & $\checkmark$ & - & - & - & DB & $\mathrm{H}$ \\
\hline Konak et al. (2006) & $\mathrm{D}$ & $\checkmark$ & - & - & & DB & $\mathrm{E}$ \\
\hline Meller, Chen, and Sherali (2007) & $\mathrm{D}$ & $\checkmark$ & - & - & - & DB & $\mathrm{E}$ \\
\hline Liu and Meller (2007) & $\mathrm{C}$ & $\checkmark$ & - & - & - & DB & MH \\
\hline Scholz, Petrick, and Domschke (2009) & $\mathrm{C}$ & $\checkmark$ & - & - & $\checkmark$ & DB & $\mathrm{H}$ \\
\hline Wong et al. (2010b) & $\mathrm{C}$ & $\checkmark$ & - & - & - & DB & MH \\
\hline Bozer and Wang (2012) & $\mathrm{C}$ & $\checkmark$ & - & - & - & DB & MH \\
\hline Kulturel-Konak (2012) & $\mathrm{C}$ & $\checkmark$ & - & - & - & $\mathrm{DB}$ & MH \\
\hline Gonçalves and Resende (2015) & $\mathrm{C}$ & - & - & - & - & DB & $\mathrm{H}$ \\
\hline Tari and Neghabi (2015) & $\mathrm{C}$ & - & - & - & - & $\mathrm{AB}$ & $\mathrm{E}$ \\
\hline Chae and Regan (2016) & $\mathrm{D}$ & $\checkmark$ & - & - & - & DB & $\mathrm{E}$ \\
\hline Paes, Pessoa, and Vidal (2017) & $\mathrm{C}$ & $\checkmark$ & - & - & - & DB & MH \\
\hline $\begin{array}{l}\text { Palomo-Romero, Salas-Morera, and García- } \\
\text { Hernández (2017) }\end{array}$ & $\mathrm{C}$ & $\checkmark$ & - & - & - & DB & $\mathrm{H}$ \\
\hline This paper & $\mathrm{C}$ & $(\checkmark)$ & - & $\checkmark$ & - & DB & $\mathrm{E}$ \\
\hline
\end{tabular}

Caption: Var. - Type of decision variables: C - Continuous or D - Discrete; Dimens. - Dimension; Irr. depart - Irregular departments; Irr. facility - Irregular facility; O.F. - Objective function: DB - Distance-based, CB - Connectivity-based, AB - Adjacency-based or SUB space utilisation based; Sol. - Solution Method: E - Exact, H - Heuristic or MH - Matheuristic;

the structure of the flexible bay structure representation. They report solving problems having up to fourteen facilities. Liu and Meller (2007) proposed a GA combining the sequence-pair representation with a mixed integer programming (MIP) model. For a given sequence-pair, the corresponding layout is determined in this hybrid approach using the linear programming relaxation of the MIP model. A tabu search algorithm for solving fixed and flexible facilities in UA-FLPs was proposed by Scholz, Petrick, and Domschke (2009). Their tabu search incorporated four types of neighbourhood moves to find better solutions. An ant colony system was proposed by Wong et al. (2010b) to solve the UA-FLP using several types of local search to improve its search performance. Later, Kulturel-Konak (2012) proposed a tabu search approach that used linear programming to determine department shapes and their locations within the bays. The proposed approach was used to solve 13 instances of different sizes from the literature and it results equalled the best results to that date. Bozer and Wang (2012) developed an hybrid approach where linear programming is used to determine the exact location of each facility and a simulated annealing algorithm is used to search for new layouts based on the graph-pair representation. Furthermore, Kulturel-Konak (2012) proposed a probabilistic tabu search (PTS) approach to solve the UA-FLP. Gonçalves and Resende (2015) proposed a biased random-key genetic algorithm with a decoder, which combines a placement strategy and a linear programming model to find the location and the dimensions of the facilities, such that the sum of the weighted distances between the centroids of the facilities is minimised. A mathematical programming model to solve the UA-FLP was developed by Tari and Neghabi (2015). In this study a new version of adjacency constraints, which provides a more flexible layout design, is proposed. Chae and Regan (2016) proposed a MIP to solve the layout design problem with two types of departments. A department's shape is given in either flexible or specified dimensions, which are denoted as Types A and B. Paes, Pessoa, and Vidal (2017) introduced two algorithmic approaches to address this problem: a basic GA, and a GA combined with a decomposition strategy via partial solution deconstructions and reconstructions. Lastly, Palomo-Romero, Salas-Morera, and García-Hernández (2017) proposed, for the first time, an Island Model Genetic Algorithm to solve the UA-FLP.

Table 1 presents the main characteristics of the various methods and procedures that have been proposed to solve the UA-FLP since the year 2000. Each model can be classified into several aspects: the type of variables representing the departments location, the decisions about the dimensions of the departments and the main considerations, such as irregular departments and facilities, department rotation, objective function and, lastly, solution method.

In terms of the layout representation, both continuous (slicing tree and the flexible bay structure) and discrete variables have been used. Each of these have advantages and disadvantages. For instance, the allocation of the departments is less constrained in the continuous representation. However, for irregular backroom shapes, discrete representation is more 


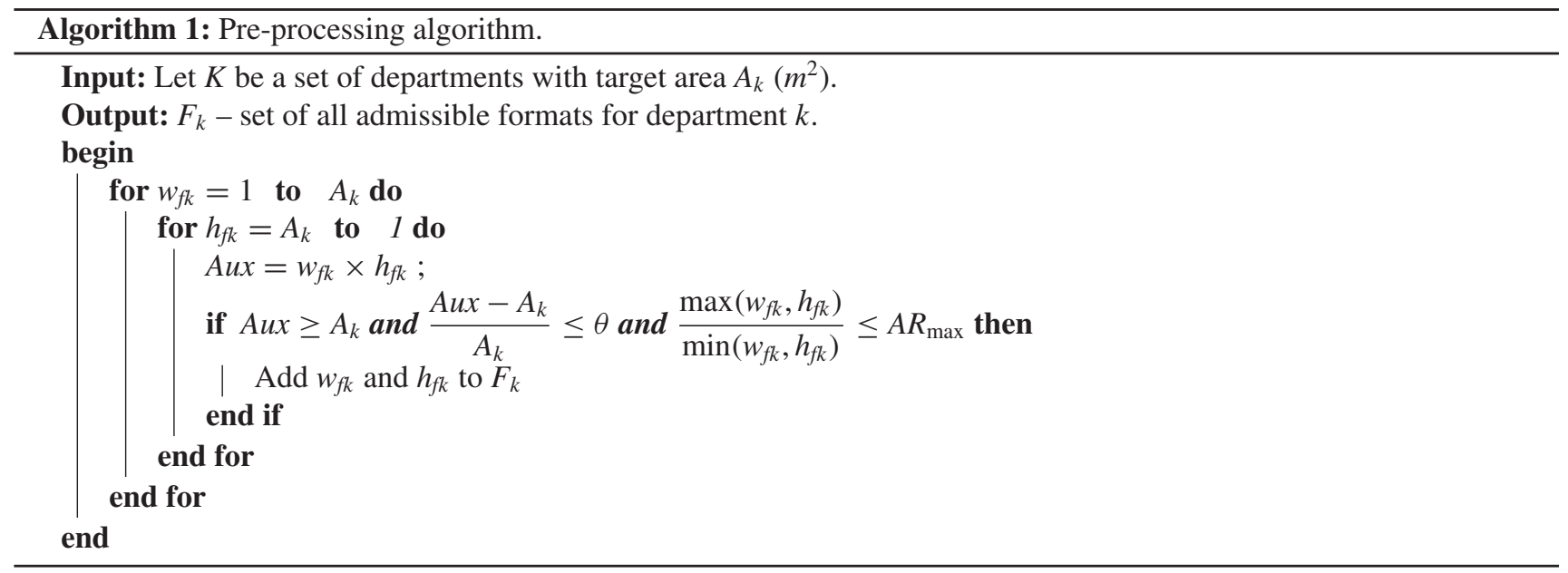

advantageous, namely in non-convex backroom shapes where the space definition is more complex using continuous variables. Nonetheless, discrete variables tend to increase the computational time. Furthermore, apart from Osman, Georgy, and Ibrahim (2003), all authors consider regular facilities. In terms of the objective function, UA-FLP models generally look at minimising the costs or flows in the facility. It is also important to notice that recent contributions in the UA-FLP field have focused in new approaches to solve the problem more efficiently rather than in extending the problem statements (e.g. incorporating constraints of flow in I/O points).

Moreover, there are other works integrating simultaneously the design of the departments and the material handling distances between departments. In Chang and $\mathrm{Ku}$ (2013) a slicing tree representation and a quadratically constrained model are combined with harmony search to develop a heuristic method for solving the problem. An ant system with slicing tree representation is also proposed for the problem in Wong et al. (2010a), good results were obtained when there was no empty space in the facility. In what concerns slicing trees, an indicator to measure the capacity of a slicing tree to generate geometrical solutions for layout problems based on the slicing tree structure is proposed in Diego-Mas et al. (2008). The indicator can predict if, by producing the proper cuts, the tree structure is able to generate layouts that satisfy the geometrical constraints considered on the items to be placed.

\subsection{Literature review discussion}

Analysing the overall grocery retail literature, there are several papers concerning sales area layout. However, they have significant limitations, such as considering the store solely as a grid layout (i.e. in a rectangular arrangement of displays along parallel aisles) when actually they have more complex layouts. Additionally, the dimensions of the departments are often assumed equal sized in the literature when, in reality, it is often not the case. Moreover, this literature stream does not acknowledge the existence of backrooms.

Regarding the literature on facility layout problems, it has focused on manufacturing enterprises and, specially, on material handling systems. In this paper, we contribute by extending the FLP to service industry, more specifically, to grocery retail store backrooms. Establishing a parallel between the UA-FLP and the BLP, in terms of the layout representation, generally backrooms can have up to 30 departments and $1000 \mathrm{~m}^{2}$. For this reason, using the discrete alternative would make the model extremely complex to solve. In terms of the objective function, the goal in the BLP resembles the FLP since it aims at minimising the total walking distances in the store. However, we also consider the sales area layout, the I/O flows between sales area and the backroom, as well as the fact that some departments should be positioned together (grouped). Lastly, the formulation proposed adds physical and operational constraints, such as a specific distribution of the storage departments in the perimeter of the backroom and the existence of a connected polygonal area linking all departments (for aisles and ambient departments).

To the best of our knowledge, the best results so far using a MIP approach were obtained by Konak et al. (2006) that reported solving optimally problems having up to 14 facilities. In this paper, the authors decided the dimensions (width and height) and the position for each department. The model that we propose is able solve optimally problems with 29 departments. However, the decisions regarding the dimensions, specifically, are decoupled from the original model. The model decides the format of each department (with associated widths and heights) instead, as well as its position. 


\section{Model development}

The solution methodology used to solve the BLP is divided into two sequential phases: pre-processing followed by the optimisation model. This methodology was chosen to simplify the optimisation model and it is based on cutting and packing problems, where a set of pieces should be placed in one (or more) large object without overlapping. In this type of problems, the set of pieces to be positioned in the big object is already defined, namely regarding its geometric dimensions. Based in this approach, we first define the possible formats (with corresponding widths and heights) of each department. Then, the mathematical model selects the best format and location for each department. In summary, the methodology proposed is divided in the following two phases:

(1) Pre-processing phase that aims to discriminate the possible backroom department formats, assuming always rectangular shapes and no rotation.

(2) Optimisation model that aims to allocate the departments in the backroom space.

Moreover, the approach uses the following assumptions:

- Backroom department dimensions are known.

- Available backroom construction area is defined, i.e. the architectural plan.

- Product flows in the backroom are known.

- Position of the categories in the sales area is known.

- Position of the access points (doors) between the sales area and backroom are known.

To describe the BLP, we consider $K=\{1, \ldots, k\}$ to be a set of unequal area backroom departments to be placed, without any overlap, on a rectangular floor space with dimensions $(W, H)$ along the $x$ - and $y$-axis, respectively, and total area of $A$. Furthermore, let $F_{k}=\left\{1, \ldots, f_{k}\right\}$ be a set of formats for each department with area $A_{f k}$ and dimensions $w_{f k} \cdot h_{f k}$.

\subsection{Pre-processing}

Since the optimal department sizes are assumed to be known, the first stage defines all the possible formats for each department. Departments must have a usable shape that allows personnel to move in the department. Therefore, we consider a constraint that defines the subset of feasible formats. An adequate aspect ratio is required for each department, which is defined as a maximum allowed value of $A R_{\max } \geq \max \{$ height, width $\} / \min \{$ height, width $\}$. Furthermore, it is admissible some slack $(\theta)$ in the department size $\left(\left(A_{f k}-A_{k}\right) / A_{k} \leq \theta\right)$, where $A_{k}$ is the target area of the department. Algorithm 1 describes the methodology used to define the formats for each department.

Figure 2 presents the possible formats for an area with $8 \mathrm{~m}^{2}$. The allowed formats are the ones that satisfy the minimum (pre-defined) area (in this case $8 \mathrm{~m}^{2}$ ) while having admissible aspect ratio values. For instance, formats 1 and 3 satisfy the minimum area, however their aspect ratio values are above the admissible upper limit defined (we considered $A R_{\max }=4$ ). On the other hand, the remaining shapes are admissible. Also, as exemplified in shape 6, formats with areas greater than the minimum required are allowed, within the slack $\theta$ (in this case $\theta=25 \%$ ).

\subsection{Optimization model}

The second phase of the solution approach consists on the optimisation model. The proposed model is inspired in the two-dimensional knapsack problem developed by Pisinger and Sigurd (2007). The objective is to find the location and the format of the departments such that the total distance is minimised. We start by describing the simplest case, which is the regular (rectangular) backroom. Nonetheless, we also show how to adapt this model to irregular backroom shapes (c.f. Section 4.2.2.). In this model, we will use the superscript $x, y$ to denote the dimensions along the $x$ and $y$ axis, respectively, considering that the overall building has its origin in its southwest corner.

Each department is characterised by its replenishment frequency, $r f_{k}$, which corresponds to the number of times that, in average, the shelf space is replenished from the backroom during the day. Let $K C$ be a subset of $K$ that only includes frozen departments and $K T$ be a subset of $K$ that only includes technical departments (i.e. that do not require I/O points to the sales area). Furthermore, the backroom layout has a set of doors $D=\{1, \ldots, d\}$. Each door is characterised by its position $\left(d_{d}^{x}, d_{d}^{y}\right)$ and by the maximum flow of products allowed to go through them per day, $f m_{d}$, in order to avoid congestion. Furthermore, the distance from door $d$ to each department in the sales area is denoted as $d s_{k d}^{x}$ and $d s_{k d}^{y}$, in $x$ and $y$ respectively.

In order to ensure that all departments are accessible and that pallet carriers can reach all departments, a minimum distance $(s)$ is guaranteed between departments. Moreover, a minimum area $(m a)$ in the centre of the backroom should be left unoccupied to store ambient-temperature merchandise, such as beverages. Lastly, we ensure that departments are 


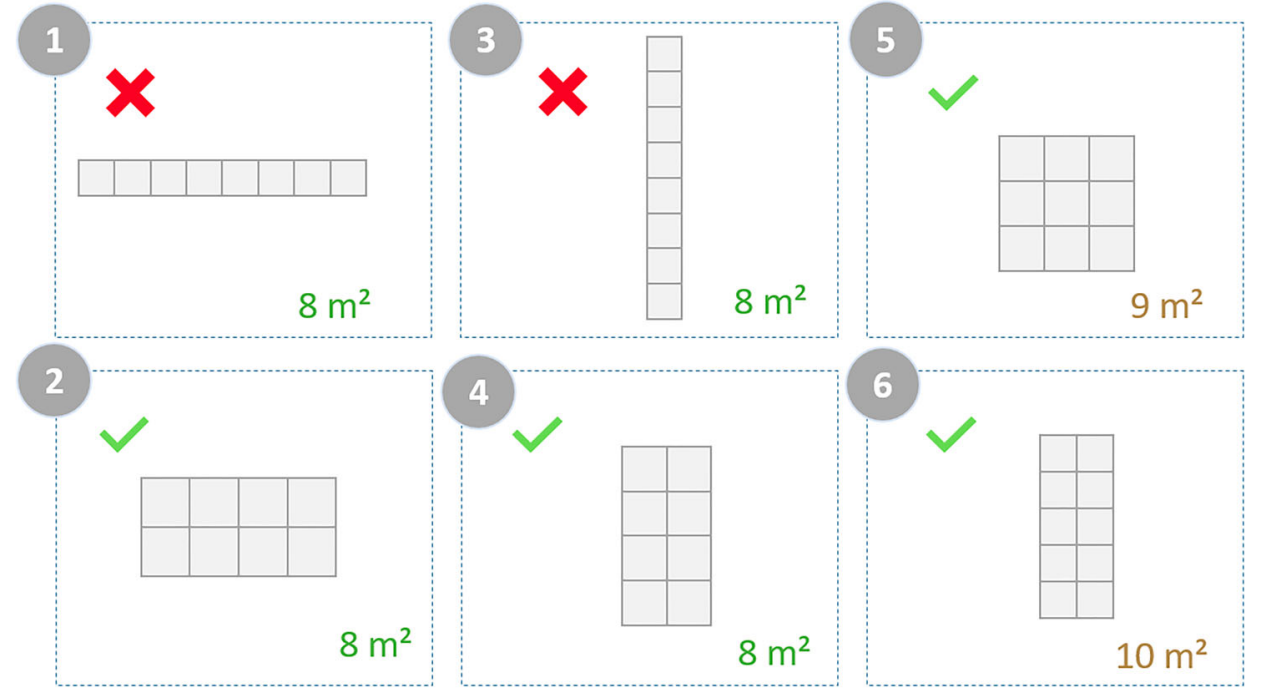

Figure 2. Representation of the possible formats for a $8 \mathrm{~m}^{2}$ department.

levelled in the $x$ and $y$-axis, using $\Delta$ as the maximum allowed difference in the departments widths, or heights, to avoid extra walls between the departments and a layout with an improved flow.

To formulate the BLP, we use decision variables $\chi_{k f}, \gamma_{k d}, x_{k}, y_{k}, \alpha_{k i}, \delta_{k i}, \sigma_{k}^{x}, \sigma_{k}^{y}, \xi_{k}^{x}$ and $\xi_{k}^{y}$ to characterise the backroom departments. Binary variables $\chi_{k f}$ are used to assign a format $f$ to a department $k$, whereas variables $\gamma_{k d}$ are used to assign departments $k$ to doors $d$. Continuous variables $x_{k}$ and $y_{k}$ define the position of departments in the $x$ and $y$-axis, respectively. Continuous variables $\sigma_{k}^{x}\left(\sigma_{k}^{y}\right)$ indicate the minimum distance in the backroom from department $k$ to door $d$ in the $x$-axis (y-axis). Moreover, continuous variables $\xi_{k}^{x}\left(\xi_{k}^{y}\right)$ indicate the sum of the interdepartmental distances in the $x$-axis ( $y$-axis) between department $k$ and the remaining. Binary variables $a_{k}, b_{k}, c_{k}$ and $d_{k}$ indicate in which of the four backroom edges the department is positioned. For instance, if $a_{k}=1$, then department $k$ is in $y=0$ and so on, sequentially in the clockwise direction. To describe the relative position of the departments, auxiliary binary variables, $\alpha_{k i}$ and $\delta_{k i}$, indicate if department $k$ is to the left or below department $i$. Continuous variables $v_{k d}^{x}\left(v_{k d}^{y}\right)$ indicate the distance from departments $k$ to door $d$ in $x$ (in $y$ ). Also, continuous variables $\omega_{k i}^{x}\left(\omega_{k i}^{y}\right)$ indicate the horizontal (vertical) distance between departments $k$ and $i$. Lastly, binary variables $\psi_{k i}^{x}\left(\psi_{k i}^{y}\right)$ indicate if departments $k$ and $i$ have the minimum distance $s$ in the $x$-axis ( $y$-axis). Appendix 1 exhibits a summary of the modelling nomenclature and in Appendix 2 a full description of each constraint formulation is presented.

\subsubsection{Regular backroom}

The proposed formulation for the regular BLP reads as follows:

$$
\text { Minimize } \sum_{k \in K \backslash\{K T\}}\left(\sigma_{k}^{x}+\sigma_{k}^{y}+\sum_{d \in D}\left(d s_{k d}^{x}+d s_{k d}^{y}\right) \cdot \gamma_{k d}\right) \cdot r f_{k}+\phi \cdot \sum_{k \in K C}\left(\xi_{k}^{x}+\xi_{k}^{y}\right)
$$

Equation (1) represents the objective function. The main goal is the minimisation of the walking distance, using the Manhattan distance as a proxy, of handling products in the store, considering both the distances in the backroom and sales area. Since the seminal paper of Montreuil (1991) that rectilinear distances are used in several UA-FLP problems, which include retail applications (Mowrey, Parikh, and Gue 2018). Furthermore, it also aims at minimising the construction distance between the frozen departments due to energy efficiency aspects. A weight $\phi$ is used to control the importance of both optimisation terms. This model is subjected to the following constraints:

Master layout constraints

$$
\begin{aligned}
& \alpha_{k i}+\alpha_{i k}+\delta_{k i}+\delta_{i k} \geq 1, \quad \forall i, k \in K: i<k \\
& \alpha_{k i}+\alpha_{i k} \leq 1, \quad \forall i, k \in K: i<k
\end{aligned}
$$




$$
\begin{aligned}
& \delta_{k i}+\delta_{i k} \leq 1, \quad \forall i, k \in K: i<k \\
& \sum_{f} \chi_{k f}=1, \quad \forall k \in K \\
& x_{k}+\sum_{f} w_{f k} \cdot \chi_{k f} \leq x_{i}+W \cdot\left(1-\alpha_{k i}\right), \quad \forall i, k \in K: i \neq k \\
& y_{k}+\sum_{f} h_{f k} \cdot \chi_{k f} \leq y_{i}+H \cdot\left(1-\delta_{k i}\right), \quad \forall i, k \in K: i \neq k \\
& x_{k} \leq W-\sum_{f}\left(w_{f k} \cdot \chi_{k f}\right), \quad \forall k \in K \\
& y_{k} \leq H-\sum_{f}\left(h_{f l} \cdot \chi_{k f}\right), \quad \forall k \in K \\
& A-\sum_{k} \sum_{f} \chi_{k f} \cdot A_{f} \geq m a \\
& \Delta \geq\left|x_{k}+\sum_{f} \chi_{k f} \cdot w_{f k}-\left(x_{i}+\sum_{f} \chi_{f i} \cdot w_{f i}\right)\right|-M \cdot\left(2-b_{i}-b_{k}\right), \quad \forall k, i \in K: i \neq k \\
& \Delta \geq\left|x_{k}-x_{i}\right|-M \cdot\left(2-d_{i}-d_{k}\right), \quad \forall k, i \in K: i \neq k \\
& \Delta \geq\left|y_{k}+\sum_{f} \chi_{k f} \cdot h_{f k}-\left(y_{i}+\sum_{f} \chi_{f i} \cdot h_{f i}\right)\right|-M \cdot\left(2-a_{i}-a_{k}\right), \quad \forall k, i \in K: i \neq k \\
& \Delta \geq\left|y_{k}-y_{i}\right|-M \cdot\left(2-c_{i}-c_{k}\right), \quad \forall k, i \in K: i \neq k
\end{aligned}
$$

Equations (2)-(4) enforce that all selected formats are arranged to the left and/or below one another. Equation (5) ensures that each department $k$ is assigned to one format $f$. Equations (6) and (7) ensure that departments do not overlap each other. Equations (8) and (9) ensure that each department $k$ is within the backroom space. Equation (10) makes sure that the chosen formats do not exceed the available backroom space and that a minimum area is left for storing merchandise at ambient temperature.

Lastly, Equations (11) and (12) guarantee that the departments are levelled, not exceeding deviations in $x$ greater than $\Delta$, to avoid extra walls. Equations (13) and (14) have the same purpose, but now considering the $y$-axis.

Door-selection constraints

$$
\begin{aligned}
\sum_{d} \gamma_{k d} & =1, \quad \forall k \in K \\
v_{k d}^{x} & =\left|d_{d}^{x}-\left(x_{k}+\sum_{f} w_{f k} / 2 \cdot \chi_{k f}\right)\right|, \quad \forall k \in K \backslash\{K T\}, d \in D \\
\sigma_{k}^{x} & \geq v_{k d}^{x}-M \cdot\left(1-\gamma_{k d}\right), \quad \forall k \in K \backslash\{K T\}, d \in D \\
v_{k d}^{y} & =\left|d_{d}^{y}-\left(y_{k}+\sum_{f} h_{f k} / 2 \cdot \chi_{k f}\right)\right|, \quad \forall k \in K \backslash\{K T\}, d \in D \\
\sigma_{k}^{y} & \geq v_{k d}^{y}-M \cdot\left(1-\gamma_{k d}\right), \quad \forall k \in K \backslash\{K T\}, d \in D \\
\sum_{k} \gamma_{k d} \cdot r f_{k} & \leq f m_{d}, \quad \forall d \in D
\end{aligned}
$$

Equation (15) ensures that each department has one door assigned. Equations (16) and (17) define the absolute distance from departments to doors, in the $x$-axis. Equations (18) and (19) define the distance to doors, in the $y$-axis. Lastly, Equation (20) defines a limit for products flow passing through each door $d$, in order to avoid congestion. 
Energy constraints

$$
\begin{aligned}
\omega_{k i}^{x} & =\left|x_{k}-x_{i}\right|, \quad \forall i, k \in K C: k \neq i \\
\xi_{k}^{x} & \geq \sum_{i} \omega_{k i}^{x}, \quad \forall k \in K C \\
\omega_{k i}^{y} & =\left|y_{k}-y_{i}\right|, \quad \forall i, k \in K C: k \neq i \\
\xi_{k}^{y} & \geq \sum_{i} \omega_{k i}^{y}, \quad \forall k \in K C
\end{aligned}
$$

Constraints (21)-(24) define the distance between the frozen departments, which is a parcel of the objective function that should be minimised. Thus, Equations (21) and (23) calculate the distances in the $x$ and $y$ axis respectively, while Equations (22) and (24) are used to calculate the total distance in the $x$ and $y$ axis respectively, between department $k$ and all other frozen departments. This ensures that these departments are placed together, reducing the construction costs.

Backroom perimeter constraints

$$
\begin{gathered}
a_{k}+b_{k}+c_{k}+d_{k} \geq 1, \quad \forall k \in K \\
H \cdot\left(1-a_{k}\right) \geq y_{k}, \quad \forall k \in K \\
W \cdot\left(1-b_{k}\right) \geqslant x_{k}, \quad \forall k \in K \\
y_{k}+\sum_{f} \chi_{k f} \cdot h_{f k} \geqslant c_{k} \cdot H, \quad \forall k \in K \\
x_{k}+\sum_{f} \chi_{k f} \cdot w_{f k} \geqslant d_{k} \cdot W, \quad \forall k \in K
\end{gathered}
$$

As previously mentioned, departments should be located in the perimeter of the backroom area. Thus, Equations (25)(29) ensure that all departments are being allocated to the edges of the backroom. Variables $a_{k}, b_{k}, c_{k}$ and $d_{k}$ correspond to one edge of the backroom.

Store operation constraints

$$
\begin{gathered}
x_{k} \leqslant x_{i}-\sum_{f} \chi_{k f} \cdot w_{f k}-s+M\left(1-\psi_{k i}^{x}\right), \quad \forall i, k \in K: k \neq i \\
x_{k} \geqslant x_{i}+\sum_{f} \chi_{i f} \cdot w_{f i}+s-M\left(1-\psi_{k i}^{x}\right), \quad \forall i, k \in K: k \neq i \\
y_{k} \geqslant y_{i}+\sum_{f} \chi_{i f} \cdot h_{f i}+s-M\left(1-\psi_{k i}^{y}\right), \quad \forall i, k \in K: k \neq i \\
y_{k} \leqslant y_{i}-\sum_{f} \chi_{k f} \cdot h_{f k}-s+M\left(1-\psi_{k i}^{y}\right), \quad \forall i, k \in K: k \neq i \\
\psi_{k i}^{x}+\psi_{k i}^{y} \geqslant a_{i}+b_{k}-1, \quad \forall i, k \in K: k \neq i \\
\psi_{k i}^{x}+\psi_{k i}^{y} \geqslant b_{i}+c_{k}-1, \quad \forall i, k \in K: k \neq i \\
\psi_{k i}^{x}+\psi_{k i}^{y} \geqslant c_{i}+d_{k}-1, \quad \forall i, k \in K: k \neq i \\
\psi_{k i}^{x}+\psi_{k i}^{y} \geqslant a_{i}+d_{k}-1, \quad \forall i, k \in K: k \neq i \\
\psi_{k i}^{x}+\psi_{k i}^{x}+\psi_{k i}^{y} \geqslant a_{i}+c_{k}-1, \quad \forall i, k \in K: k \neq i \\
\psi_{k i}^{x}+\psi_{k i}^{y}+\psi_{k i}^{y} \geqslant b_{i}+d_{k}-1, \quad \forall i, k \in K: k \neq i
\end{gathered}
$$

To ensure smooth in-store operations in the backroom it is necessary to ensure the existence of a connected polygonal area that links all departments. This area serves both as an aisle to move merchandise around, and as a storage area for ambient products and promotions. Constraints (30)-(39) ensure the existence of the connected polygonal area linking all departments, and that the aisles have at least $s$ space to move the products (the size of a pallet truck was considered). In Appendix 2 a full description of this set of constraints is presented. 


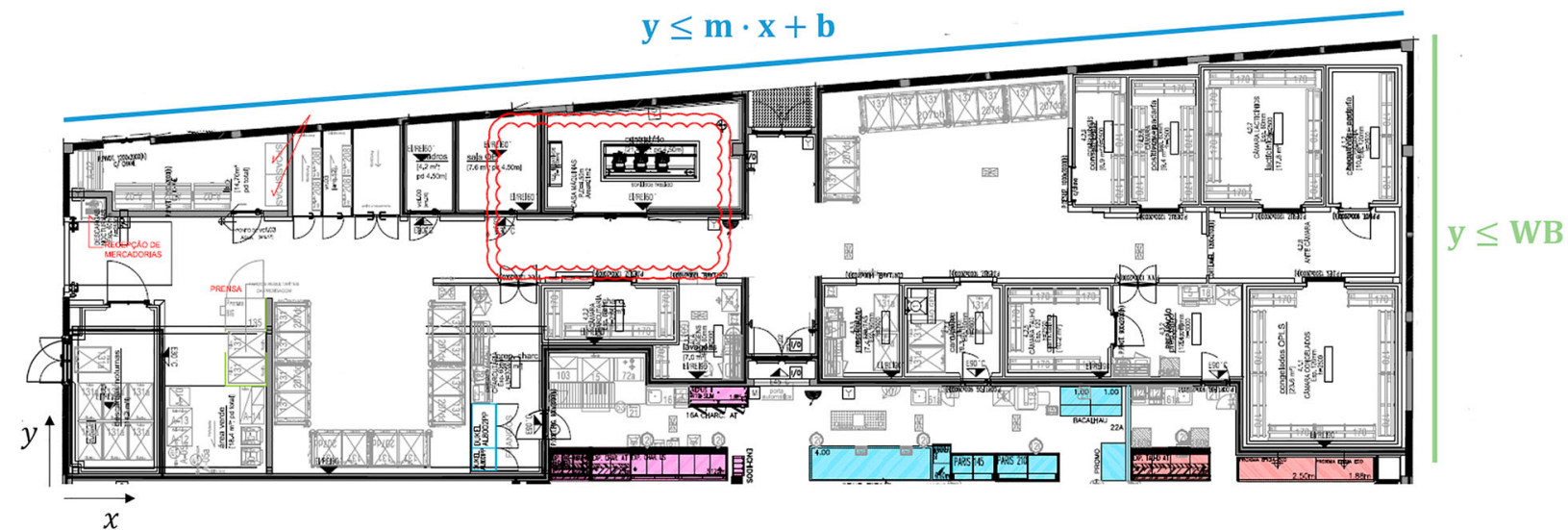

Figure 3. Example of an irregular backroom.

Variables domain

$$
\begin{aligned}
& \chi_{k f}, \gamma_{k d}, \alpha_{k i}, \delta_{k i}, \psi_{k i}^{x}, \psi_{k i}^{y}, a_{k}, b_{k}, c_{k}, d_{k} \in\{0,1\} \\
& x_{k}, y_{k}, v_{k d}^{x}, v_{k d}^{y}, \sigma_{k}^{x}, \sigma_{k}^{y}, \xi_{k}^{x}, \xi_{k}^{y}, \omega_{k i}^{x}, \omega_{k i}^{y} \in \mathbb{R}^{+}
\end{aligned}
$$

These last constraints define the domain of the variables.

\subsubsection{Irregular backroom}

In case of having irregular backroom shapes, constraints (6)-(9) and (25)-(32) could have to be adapted to the new shape. In Figure 3, an example of an irregular backroom is illustrated. In this layout, the top edge is inclined, as an example. This section describes how to cope with the irregular shape presented in Figure 3.

When one or more of the edges have a slope different than zero, it affects the 'admissible region' for placing the backroom departments. The area in which the departments can be allocated is defined by former Equations (8) and (9). In this example, only former Equation (9) is adapted to Equation (42) to cope with the irregular shape illustrated in this example. Here, $m$ is the slope of the wall, that previously was zero, and $b$ is the intersection in the $y$-axis.

$$
y_{k}+\sum_{f} \chi_{k f} \cdot h_{f k}-m \cdot x_{k}-b \leq 0, \quad \forall k
$$

Furthermore, since the departments should be allocated in the perimeter of the backroom, former Equation (28) is now transformed into Equation (43), that copes with this irregularity. It is important to notice that, since the slope of the line is positive, the reference vertex that should be in contact with this line is the top-left vertex. However, if the slope was negative, it would have been the top-right vertex instead.

$$
y_{k}+\sum_{f} \chi_{k f} \cdot h_{f k} \geqslant\left(b+m \cdot x_{k}\right)-M \cdot\left(1-c_{k}\right), \quad \forall k
$$

\section{Computational experiments}

This section presents the numerical results of our optimisation approach and provides managerial insights regarding the BLP. First, we present the instances used to test the model proposed to solve the BLP. Then, we quantify the savings obtained by using the model proposed when compared with a baseline solution (provided by the case study retailer). Afterwards, we test four different models in order to unveil the heuristic beyond the current design process. Lastly, we provide an illustrative example of the methodology followed.

All numerical tests were conducted on Intel Xeon E5-2687 @3.100 GHz CPU and 64 GB of physical memory. 
Table 2. Instance characterisation.

\begin{tabular}{|c|c|c|c|c|c|c|c|c|}
\hline Store & \# Dpts & \# Frozen dpt & \# Ref dpt & \# Doors & \# Formats & Irregular & N.R. area & Area $\left(m^{2}\right)$ \\
\hline Store A & 15 & 2 & 12 & 1 & 26 & yes & $15 \%$ & 1843 \\
\hline Store B & 15 & 2 & 7 & 1 & 43 & - & $29 \%$ & 1247 \\
\hline Store C & 17 & 2 & 10 & 1 & 41 & yes & $50 \%$ & 1332 \\
\hline Store D & 17 & 3 & 13 & 2 & 52 & - & $39 \%$ & 1794 \\
\hline Store E & 20 & 3 & 12 & 1 & 41 & - & $28 \%$ & 1177 \\
\hline Store F & 20 & 3 & 13 & 2 & 50 & - & $27 \%$ & 2115 \\
\hline Store G & 26 & 2 & 13 & 2 & 73 & yes & $45 \%$ & 2269 \\
\hline Store H & 29 & 3 & 13 & 2 & 10 & - & $20 \%$ & 2722 \\
\hline
\end{tabular}

Table 3. Computational results for the original model.

\begin{tabular}{lccccccc}
\hline Store & \#Variables & \#Constraints & Model O.F. & Time (s) & Gap (\%) & Real O.F. & Deviation (\%) \\
\hline Store A & 3465 & 5124 & 2536,7 & 14,400 & $1,8 \%$ & 3014,5 & $25,8 \%$ \\
Store B & 4694 & 6280 & 2130 & 20 & $0,0 \%$ & 3504,5 & $37,2 \%$ \\
Store C & 5837 & 7367 & 3012,9 & 5520 & $0,0 \%$ & 3797,5 & $20,7 \%$ \\
Store D & 6738 & 9682 & 2091 & 368 & $0,0 \%$ & 4227 & $45,4 \%$ \\
Store E & 7822 & 10,947 & 3533,1 & 14,400 & $2,1 \%$ & 4662,3 & $24,2 \%$ \\
Store F & 8781 & 10,971 & 3323,9 & 6960 & $0,0 \%$ & 4632,6 & $28,2 \%$ \\
Store G & 14,477 & 19,445 & 2901,5 & 370 & $0,0 \%$ & 4283,9 & $32,3 \%$ \\
Store H & 8781 & 10,971 & 2502 & 4840 & $0,0 \%$ & 4147,7 & $38,7 \%$ \\
\multicolumn{2}{l}{ Avg regular shapes } & & & 5317,2 & $0,4 \%$ & $34,5 \%$ & $26,3 \%$ \\
\multicolumn{2}{l}{ Avg irregular shapes } & & & 6763,3 & $0,6 \%$ & & \\
\hline
\end{tabular}

\subsection{Experimental set-up}

To evaluate the performance and the capabilities of the model proposed, we performed a series of computational experiments. As smaller store formats (proximity stores) are growing more rapidly, they are the focus of our study (Nielsen 2015).

The data to build our instance set was provided by a large food retailer operating in Europe. The model was tested using eight layouts of existent convenience stores, which were provided by the case study company. The sample of stores represents all convenience stores in a way that it includes several locations, backroom sizes and shapes.

The characterisation of the instances is presented in Table 2. The main characteristics of the instances are the number of departments (total, frozen and refrigerated departments), number of doors (I/O points), number of possible formats, the shape of the backroom (regular or irregular), the non-refrigerated area (in \%) ${ }^{1}$ and, lastly, the store total area (in $\mathrm{m}^{2}$ ). In terms of departments, there are three different types: storage, preparation and technical departments. From these, only storage and preparation departments influence the walking distance in the store. However, since all departments must be placed in the backroom, technical departments add computational complexity.

\subsection{General results}

Table 3 summarises the results for applying the approach described on Section 4 to the several instances while using a general purpose solver (CPLEX). This table describes the number of variables, number of constraints, objective function (O.F.) value, running time (in seconds), gap (for the cases where the solver could not find the optimal solution within the time limit) for each store, the objective function value of the stores' actual layout and, lastly, the deviation between the two objective function values.

The time limit was set for four hours $(14,400 \mathrm{~s})$, which is appropriate since this is a strategic problem. Optimal solutions were found for six out of the eight instances analysed. Nonetheless, these unsolved instances had a gap of only $2 \%$. The optimal solution for Store A was found after 60,015 s with value 2489.1, and the optimal solution for Store E was found after 158,130 with value 3507.1. Store A has the smallest percentage of non-refrigerated area, and Store E, is the store with the smallest area, we speculate that these can be the reasons for the difficulty of the solver to find the optimal solution. The computational efficiency depends on the combination of the number of departments, possible shape formats and I/O points, which directly impact the number of variables. As these increase, the complexity and solving time of the problem increases 
as well. On the other hand, when the non-refrigerated area (in \%) increases, the simpler the problem becomes since there is more flexibility to allocate the departments in the backroom. Furthermore, the irregularity of the backroom also adds complexity to the problem.

In the objective function, the $\phi$ parameter is used to assign a weight to the total distance between the frozen departments. In these computational tests, $\phi=1$ was considered, so that the objective function would reflect the minimisation of the walking distances. The model allows the decrease of the walking distance by $30 \%$ on average, which creates better conditions for the store employees as well as an opportunity to create better service (lower out-of-shelf situations) to customers. Ultimately, this might result in higher store sales, customer satisfaction and lower personnel costs. Analysing in detail, it is possible to observe that the model is able to create more improvements for regular stores than for irregular stores. The store with the largest deviation is store $\mathrm{D}$, with a $45,4 \%$ deviation between the current solution and the one proposed by the model. This happens because the store departments with higher replenishment frequencies are located further away from the $\mathrm{I} / \mathrm{O}$ points to the sales area in the real layout.

As mentioned in the Literature Review section, to the best of our knowledge, the best results so far using a MIP model were obtained by Konak et al. (2006) considering 14 departments considering flexible bay structures, which is a continuous layout representation allowing the departments to be located only in parallel bays with varying widths. The width of each bay depends on the total area of the departments in the bay and bays are bounded by straight aisles on both sides, restricting the possible layout configurations. The MIP model proposed for the BLP was able to solve an instance with 29 departments, although the MIP model does not (fully) decide the dimensions of the departments, and considers other important practical constraints such as door selection, and irregular backroom facilities.

\subsection{Unveiling the practitioners heuristics}

Nowadays, the design of the backroom areas is mainly established empirically, based on the perception of the architects inspired on similar stores.

What often happens is that store layouts reflect the subjectivity and preferences of each designer. Hence, the criteria used to allocate departments in the backroom is not clear. In this analysis we attempt to grasp the heuristic behind the design process currently followed in practice, i.e. what are the current objective and constraints being used besides the space (physical) constraints. Thus, four variations of the model previously defined were tested (scenario 1-4). Each of these scenarios neglect one important aspect considered in the original model. These aspects were selected based on the discussions with the company's designers while framing the problem. The four scenarios are as follows:

- Scenario 1: This scenario does not consider the location of the product categories in the sales area in the objective function. Therefore, it only aims at minimising the distance within the backroom.

- Scenario 2: This scenario does not consider energy efficiency issues. Therefore, it does not aim at minimising the distance between frozen departments.

- Scenario 3: This scenario ignores replenishment frequencies for the different storage departments. Thus, in this scenario, all departments are considered similar in terms of how many times they are visited daily by an employee.

- Scenario 4: This last scenario neglects the flow in the doors, i.e. the solution of this model ignores the congestion in the doors and, therefore, does not attempt to distribute the flow of merchandise as it is actually desired in practice.

Scenarios 1, 2 and 3 are obtained by modifying the objective function whereas the last one is achieved by removing Equation (20) that ensures flow balance in the doors by setting a limit. Appendix 3 provides further details on how to model each of these scenarios.

These scenarios have the goal of understanding what is currently the design strategy of the case study company, by analysing the closest objective function value to the real layout and the closest department locations to the current positions. Thus, it is possible to understand the aspects that designers are considering and what they are neglecting. With this analysis, we aim to provide managerial insights to help designers make more structured decisions in the future.

\subsubsection{Comparison results}

Table 4 presents the results for each pair scenario-store analysed. When aggregating the results by scenario and analysing the deviation of the objective function, we realise that the mean deviations are, in ascending order, $21,8 \%$ for Scenario 3 , 26,9\% for Scenario 4, 29,6\% for Scenario 2, and, lastly, 58,3\% for Scenario 1. Interestingly, the same order happens to hold when analysing the deviation between the position of the departments in the different scenarios and in reality. Thus, it is plausible to conclude that the closest scenario to the real heuristic used by practitioners is Scenario 3 (which does not consider replenishment frequencies), followed by Scenario 4 (which does not consider congestion at the I/O points). Also, 
Table 4. Scenario analysis results.

\begin{tabular}{|c|c|c|c|c|c|c|c|}
\hline Scenario & Store & O.F. & Time & Gap (\%) & Real O.F. & Deviation O.F. & Deviation Loc. \\
\hline & Store A & 673 & 14,400 & $7,1 \%$ & 1197,7 & $43,8 \%$ & 26,31 \\
\hline & Store B & 536 & 18 & $0,0 \%$ & 2113,8 & $66,6 \%$ & 37,42 \\
\hline & Store C & 556,5 & 5519 & $0,0 \%$ & 1152 & $51,7 \%$ & 30,86 \\
\hline & Store D & 634,9 & 14,400 & $1,2 \%$ & 1717,7 & $61,0 \%$ & 68,50 \\
\hline & Store E & 1058,5 & 14,400 & $13,0 \%$ & 2190,7 & $46,7 \%$ & 35,20 \\
\hline & Store F & 728,1 & 14,400 & $5,6 \%$ & 2041,5 & $64,3 \%$ & 13,8 \\
\hline & Store G & 711,7 & 14,400 & $5,1 \%$ & 2049,9 & $63,3 \%$ & 128,35 \\
\hline & Store H & 640,5 & 8940 & $0,0 \%$ & 1998,5 & $68,0 \%$ & 58,86 \\
\hline \multirow[t]{9}{*}{ Avg } & & & & & & $58,3 \%$ & 399,30 \\
\hline & Store A & 2490 & 14,400 & $1,8 \%$ & 3014,5 & $17,4 \%$ & 10,99 \\
\hline & Store B & 2130 & 20 & $0,0 \%$ & 3707,8 & $42,6 \%$ & 40,10 \\
\hline & Store C & 3005,5 & 4800 & $0,0 \%$ & 3492,2 & $13,9 \%$ & 25,10 \\
\hline & Store D & 2083,35 & 288 & $0,0 \%$ & 4127,2 & $44,5 \%$ & 19,20 \\
\hline & Store E & 3521,6 & 14,400 & $2,3 \%$ & 4660,1 & $24,4 \%$ & 47,20 \\
\hline & Store F & 3321 & 987 & $0,0 \%$ & 4628,7 & $28,3 \%$ & 11,30 \\
\hline & Store G & 2897,5 & 468 & $0,0 \%$ & 4249,9 & $31,8 \%$ & 114,72 \\
\hline & Store H & 2478,5 & 940 & $0,0 \%$ & 3629,1 & $31,7 \%$ & 101,50 \\
\hline \multirow[t]{9}{*}{ Avg } & & & & & & $29,9 \%$ & 370,12 \\
\hline & Store A & 285 & 14,400 & $11,3 \%$ & 287,8 & $2,0 \%$ & 25,23 \\
\hline & Store B & 155,5 & 520 & $0,0 \%$ & 261,1 & $35,2 \%$ & 32,20 \\
\hline & Store C & 317,4 & 14,400 & $0,3 \%$ & 390,8 & $18,1 \%$ & 29,05 \\
\hline & Store D & 260 & 14,400 & $4,1 \%$ & 460,1 & $34,3 \%$ & 18,10 \\
\hline & Store E & 331,2 & 14,400 & $9,0 \%$ & 399,94 & $16,7 \%$ & 51,48 \\
\hline & Store F & 295,3 & 14,400 & $3,7 \%$ & 388,5 & $22,1 \%$ & 11,40 \\
\hline & Store $\mathrm{G}$ & 340,8 & 14,400 & $1,7 \%$ & 390,8 & $11,6 \%$ & 102,47 \\
\hline & Store H & 350,3 & 3280 & $0,0 \%$ & 602 & $34,6 \%$ & 45,78 \\
\hline \multirow[t]{9}{*}{ Avg } & & & & & & $21,8 \%$ & 315,71 \\
\hline & Store A & 2490 & 14,400 & $1,8 \%$ & 3014,5 & $17,4 \%$ & 33,81 \\
\hline & Store B & 2130 & 19 & $0,0 \%$ & 3707 & $37,5 \%$ & 35,30 \\
\hline & Store C & 3012,95 & 5160 & $0,0 \%$ & 3504,5 & $14,0 \%$ & 20,50 \\
\hline & Store D & 2291 & 432 & $0,0 \%$ & 4002,5 & $38,8 \%$ & 28,90 \\
\hline & Store E & 3533 & 14,400 & $1,8 \%$ & 4662,3 & $24,2 \%$ & 32,40 \\
\hline & Store F & 3136,9 & 6480 & $0,0 \%$ & 4147,2 & $24,4 \%$ & 20,50 \\
\hline & Store G & 2633,5 & 359 & $0,0 \%$ & 3681,8 & $28,5 \%$ & 125,24 \\
\hline & Store H & 2283,5 & 6480 & $0,0 \%$ & 3409,1 & $33,0 \%$ & 23,60 \\
\hline Avg & & & & & & $26,9 \%$ & 320,26 \\
\hline
\end{tabular}

Scenario 1 is the one that is more distant to the current practice. The combination of scenarios 3 and 4 was also tested, but the deviations obtained were higher than for scenario 3 . These results are relevant from a practical point of view, since they help to conjecture what has been considered and ignored when designing backrooms. Hence, we hypothesise that designers have been neglecting the replenishment frequencies and door assignment, and have been considering the location of the departments in the sales area. This can occur for several reasons, namely the lack of the architects' knowledge in relation to in-store operations (e.g. products flow, replenishment frequencies).

In terms of model efficiency, it can be observed that when the replenishment frequencies and sales area locations are removed from the objective function, the models perform worse.

\subsubsection{Illustrative example}

In this section we illustrate the methodology used and the results obtained for Store $\mathrm{G}$ in the previous section (original model and hypothetical scenarios). Store G (Figure 4) is characterised by 28 departments of which 2 are departments to store frozen goods, 8 are departments to store refrigerated goods, 3 are preparation areas and 13 are technical departments.It is important to mention that in this example, there are departments that overlay each other (e.g. department 15 e overlayed by department 16 and 17). However, this type of layout is not considered in the mathematical formulation, since practitioners do rarely consider this layout, and it should be avoided mainly for congestion reasons. This type of layout is not convenient in terms of in-store operations since the access to these departments is only possible by crossing other departments. Further details regarding this store are provided in Appendix 4 (Figure 4). 


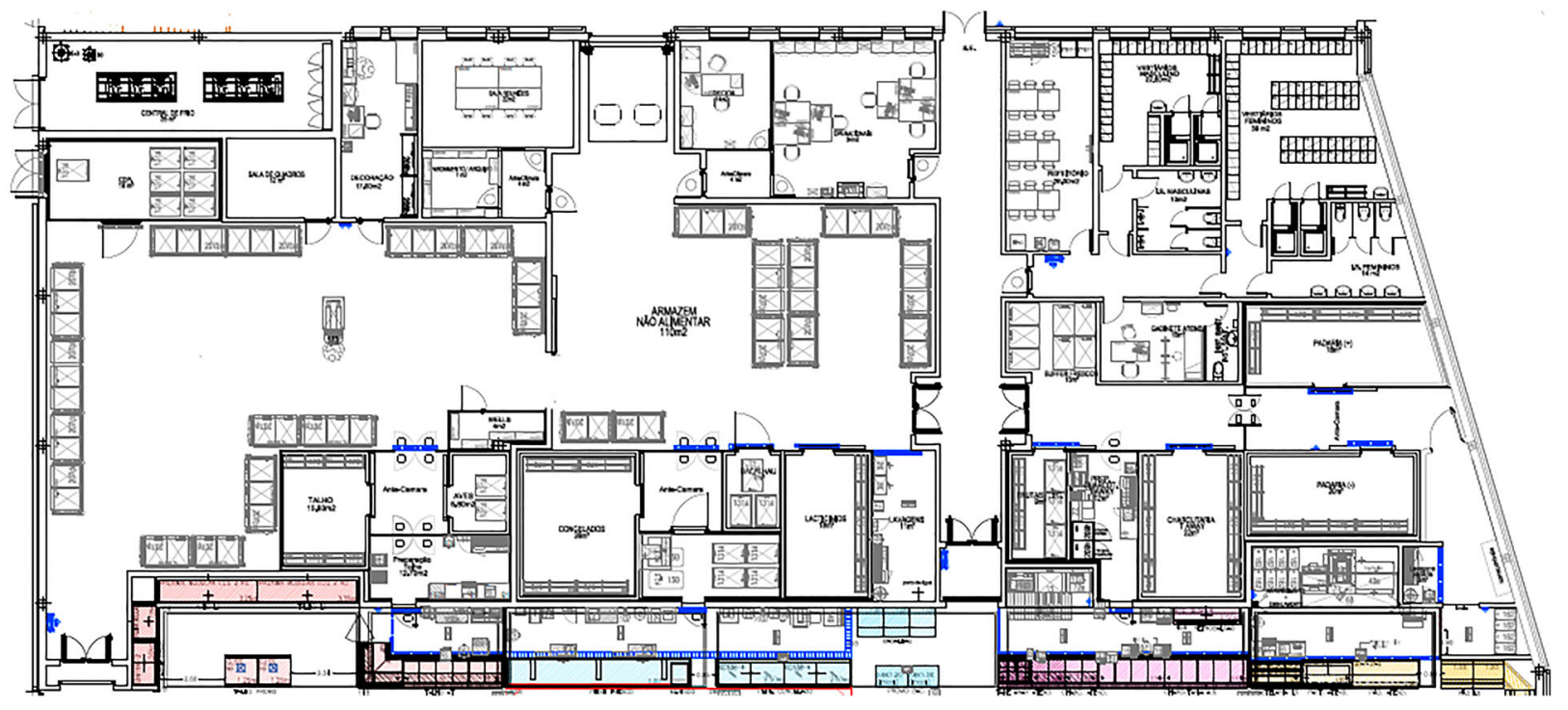

Figure 4. Real layout of a backroom.

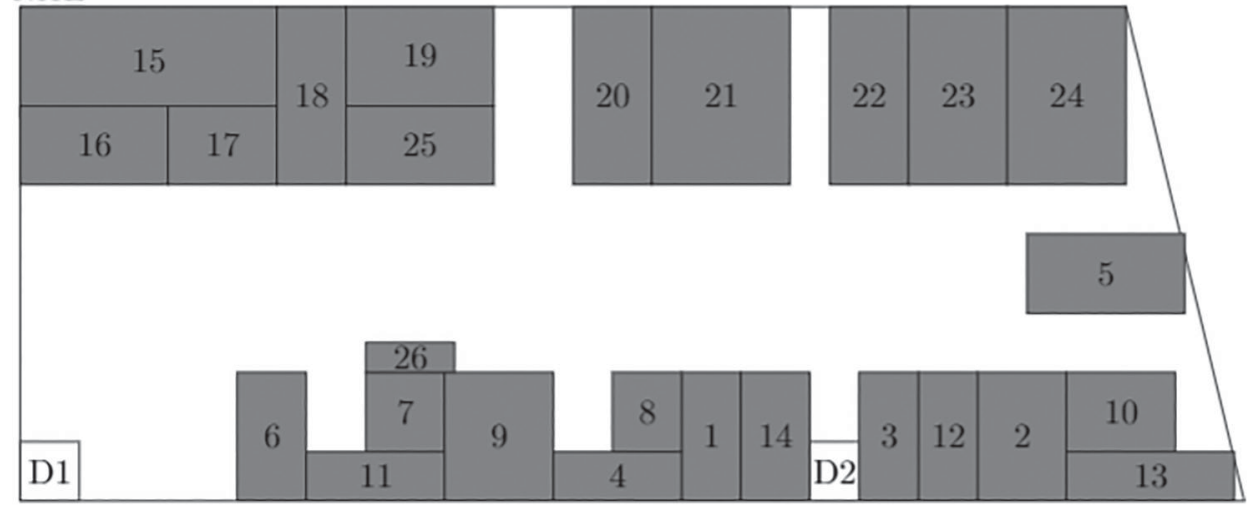

Figure 5. Model abstraction of the real layout.

The original model places the departments in a way that minimises the total walking distance in the store, considering the sales area location, replenishment frequencies, sales area layout and energy aspects (Figure 6). Departments around door 1 (door to the left) are departments located in the left part of the sales area. The same happens with departments around door 2 (right part of the sales area). Furthermore, departments 3, 4, 6, 11, 12 and 13 are the ones with the highest replenishment frequencies and that is the reason why they are close to the doors, these are meat, fish, fruits \& vegetables and preparation areas. By analysing the real layout (Figure 5), we can conclude that this aspect has not been considered in the actual backroom layout. Furthermore, we can see that in the real layout departments are not as close to door 1 as they should, in order to minimise the walking distance. Thus, product flows are not balanced, as $75 \%$ of products flows are assigned to door 2. Moreover, frozen departments 9 and 10 are next to each other in order to minimise heat loss. The departments that were positioned in the top of the backroom are the technical and social departments, that do not require to be near the sales area.

In the first scenario (without considering the location of the product categories in the sales area) the model only considers the distances in the backroom (Figure 7). Therefore, the backroom layout presents a different arrangement of departments. The departments that before were closer to the corresponding locations in sales area are now in other positions. Therefore, the replenishment frequencies only affect the walking distances within the backroom. Thus, departments with higher replenishment frequencies remain next to the doors. Furthermore, frozen departments remain together. In the scenario where frozen departments are not obliged to be positioned adjacent to each other (scenario 2), we see in the solution (Figure 8) that departments 9 and 10 are, in fact, separate which would lead to an increase in the energy costs. The remaining aspects are still considered. In the scenario where replenishment frequencies were neglected, we see a different arrangement from 


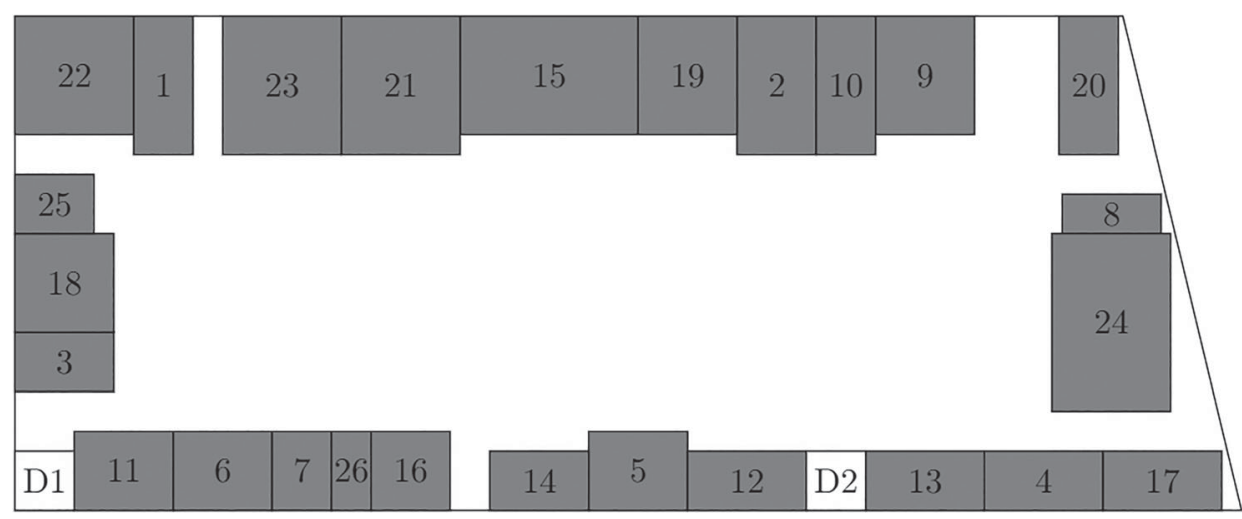

Figure 6. Solution for scenario 0.

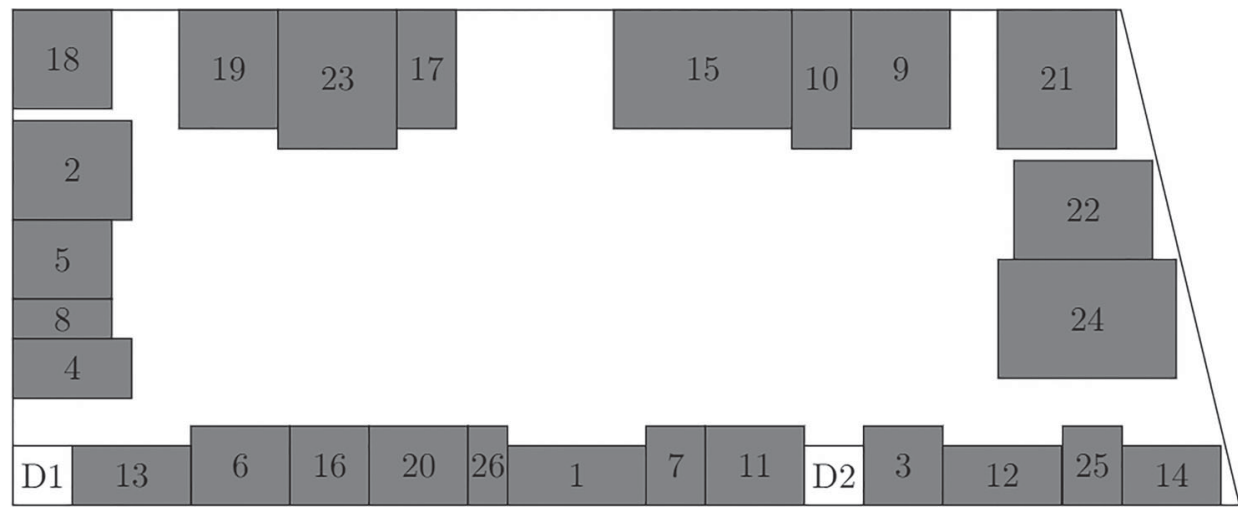

Figure 7. Solution for scenario 1.

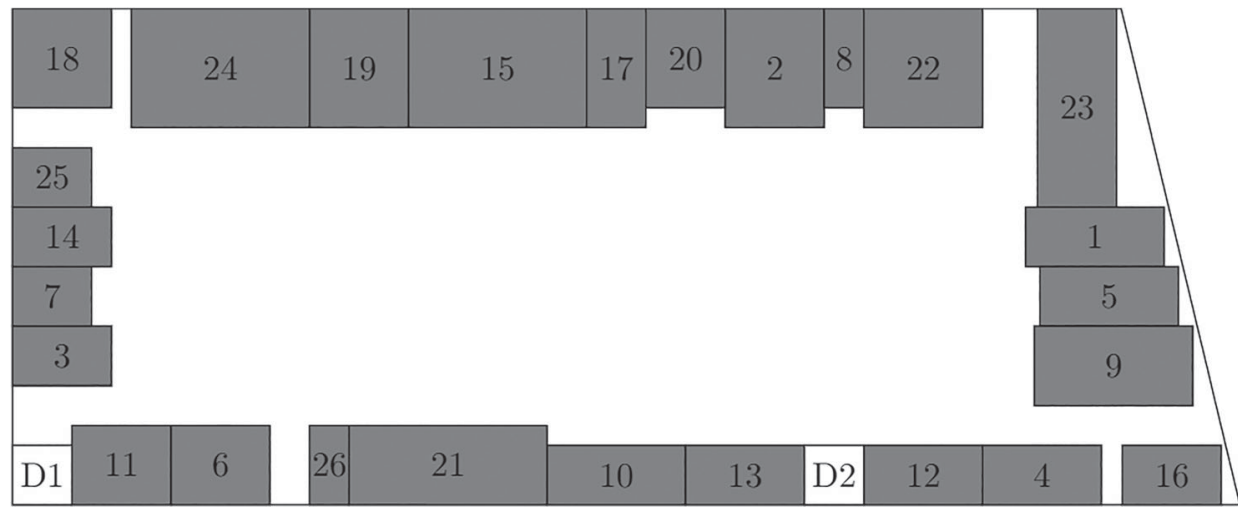

Figure 8. Solution for scenario 2 .

all the above (Figure 9). Here, the departments are allocated to the right doors, however, the order in which the departments are located does not represent the frequency in which departments are visited by employees during the day. For instance, departments 6 and 13 are not as close to the doors as in the other solutions. In the fourth scenario, the flow passing through each door is not considered (Figure 10). Therefore, we see that door 2 (in the right) has more departments assigned than door 1 (in the left), which would cause congestion. As Table 4 indicates, Scenario 3 is the one that best represents the current heuristic used by designers to design backrooms. In the specific case of store G, the deviation is of 11,6\%. 


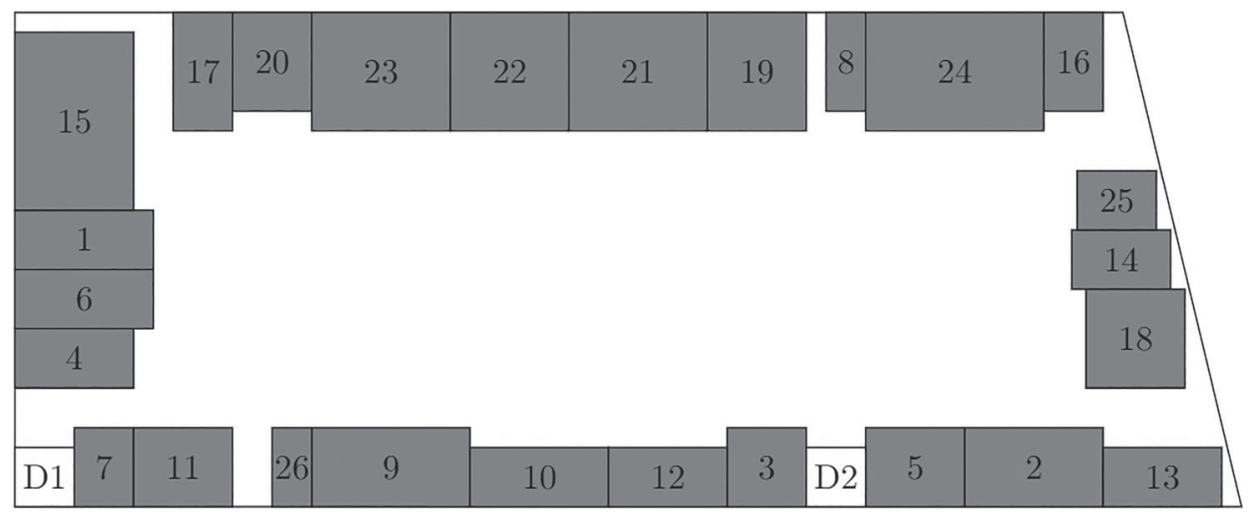

Figure 9. Solution for scenario 3.

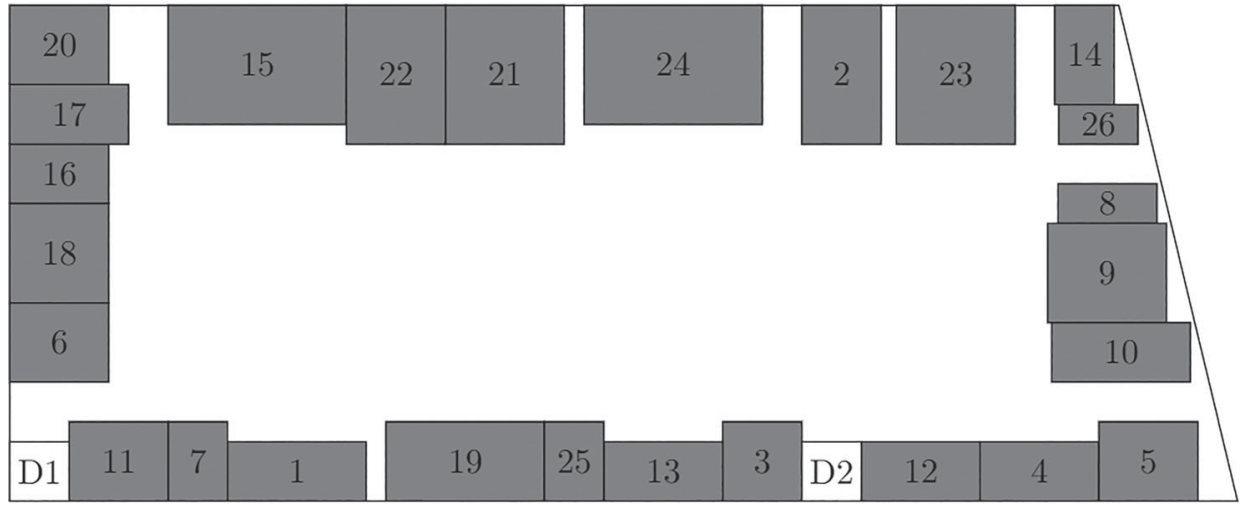

Figure 10. Solution for scenario 4.

\section{Concluding remarks}

In this paper we present a mathematical programming model for the BLP, applied to the grocery retail context. In this problem, a set of unequal area rectangular facilities with a given area requirements have to be placed, without overlapping, on the backroom, which can have rectangular or irregular shapes. The aim of the problem is to minimise the walking distances in the stores. The model is tested on a sample of existing stores.

The BLP is a strategic problem, which admits long running times. Within the time limit of four hours, optimal solutions were found for six out of the eight instances analysed. Nonetheless, these instances had a gap of only $2 \%$. Since the BLP is a strategic problem, if an optimal solution is required, running times should be increased. The objective function mostly reflects the walking distance in the store, and by using the model proposed the walking distance in the store decreases approximately $30 \%$.

Besides the original model, four scenarios were created, each neglecting a different designing aspect. By comparing these scenarios with the real layouts, it was possible to estimate what is the current designing heuristic beyond the current layouts. According to this analysis, designers are currently neglecting the replenishment frequencies as well as congestion in $\mathrm{I} / \mathrm{O}$ points.

The proposed model fills a gap in the literature, since this problem has not yet been tackled. Furthermore, it adds value in practice since it is a cornerstone in enabling architects to have a systematic and standard methodology to design backroom layouts, considering in-store operations and products flow. It also allows to decrease the backroom planning times and resources needed for this task. After obtaining the solution of the model, it might be necessary the intervention of the architects and engineers to make some adjustments and define additional technical issues. Nonetheless, it frees them of the greater task of allocating the departments, which usually takes days. Stores may also benefit from the new proposed layouts in terms of increasing customer service. Shorter walking distance lead to less out-of-shelf situations (since employees are more willing to replenish the shelves from the backroom), additional time for employees to perform other tasks (e.g. supporting customers) and, lastly, provides better conditions to employees (e.g. less injuries caused by carrying heavy merchandise for long distances). 
As future work, it would also be interesting to adapt the model to non-convex shapes, which exist in urban stores, test the model in other settings, such as consumer electronics retail and, lastly, integrate the layouts of the sales and backroom areas.

\section{Notes}

1. The non-refrigerated area (in \%) corresponds to the quotient between the free area - available space (in $\mathrm{m}^{2}$ ) left when the required storage area of frozen and refrigerated departments is removed from the total backroom area - and the total backroom area.

2. Constrained $a_{k}+b_{k}+c_{k}+d_{k}=1$ was also tested, however the computational results did not improve.

\section{Disclosure statement}

No potential conflict of interest was reported by the authors.

\section{Funding}

This work is financed by the North Portugal Regional Operational Programme (NORTE 2020), under the PORTUGAL 2020 Partnership Agreement, and through the European Regional Development Fund (ERDF), within the project 'NORTE-01-0145-FEDER-000020'. The second author was also financed by the ERDF - European Regional Development Fund through the Operational Programme for Competitiveness and Internationalisation - COMPETE 2020 Programme and by National Funds through the Portuguese funding agency, Fundação para a Ciência e a Tecnologia within project 'POCI-01-0145-FEDER-029609'.

\section{References}

Aloysius, G., and D. Binu. 2013. "An Approach to Products Placement in Supermarkets Using Prefixspan Algorithm.” Journal of King Saud University - Computer and Information Sciences 25 (1): 77-87.

Altuntas, S., and S. Altuntas. 2017. "A Novel Approach Based on Utility Mining for Store Layout: a Case Study in a Supermarket." Industrial Management \& Data Systems 117 (2): 304-319.

Anjos, M. F., and M. V. Vieira. 2017. "Mathematical Optimization Approaches for Facility Layout Problems: The State-of-the-Art and Future Research Directions.” European Journal of Operational Research 261 (1): 1-16.

Armour, G. C., and E. S. Buffa. 1963. "A Heuristic Algorithm and Simulation Approach to Relative Location of Facilities." Management Science 9 (2): 294-309.

Barbosa-Povoa, A. P., R. Mateus, and A. Q. Novais. 2001. “Optimal Two-Dimensional Layout of Industrial Facilities.” International Journal of Production Research 39 (12): 2567-2593.

Bianchi-Aguiar, T., E. Silva, L. Guimarães, M. A. Carravilla, and J. F. Oliveira. 2018. "Allocating Products on Shelves Under Merchandising Rules: Multi-Level Product Families with Display Directions.” Omega 76: 47-62.

Bijvank, M., and I. F. Vis. 2011. "Lost-Sales Inventory Theory: A Review.” European Journal of Operational Research 215 (1): 1-13.

Botsali, A. R. 2007. "Retail Facility Layout Design.” PhD thesis, Texas A \& M University.

Bozer, Y. A., and C.-T. Wang. 2012. "A Graph-Pair Representation and Mip-Model-Based Heuristic for the Unequal-Area Facility Layout Problem." European Journal of Operational Research 218 (2): 382-391.

Chae, J., and A. C. Regan. 2016. "Layout Design Problems with Heterogeneous Area Constraints." Computers \& Industrial Engineering 102: 198-207.

Chandra, C., and J. Grabis. 2008. "Inventory Management with Variable Lead-Time Dependent Procurement Cost." Omega 36 (5): 877-887.

Chang, M.-S., and T.-C. Ku. 2013. "A Slicing Tree Representation and QCP-Model-Based Heuristic Algorithm for the Unequal-Area Block Facility Layout Problem.” Mathematical Problems in Engineering 2013: 853586.

Cil, I. 2012. "Consumption Universes Based Supermarket Layout Through Association Rule Mining and Multidimensional Scaling." Expert Systems with Applications 39 (10): 8611-8625.

Diego-Mas, J., M. Santamarina-Siurana, V. Cloquell-Ballester, and J. Alcaide-Marzal. 2008. "Slicing Tree's Geometric Potential: An Indicator for Layout Problems Based on Slicing Tree Structure.” International Journal of Production Research 46 (4): $1071-1087$.

Drira, A., H. Pierreval, and S. Hajri-Gabouj. 2007. "Facility Layout Problems: A Survey." Annual Reviews in Control 31 (2): $255-267$.

Dunne, P. M., and R. F. Lusch. 1999. Retailing. Fort Worth, TX: The Dryden Press.

Fisher, M. 2004. “To Me it's a Store. to You it's a Factory.” International Commerce Review: ECR Journal 4 (2): 9.

Gonçalves, J. F., and M. G. Resende. 2015. "A Biased Random-Key Genetic Algorithm for the Unequal Area Facility Layout Problem." European Journal of Operational Research 246 (1): 86-107.

Hübner, A. H., and H. Kuhn. 2012. "Retail Category Management: State-of-the-Art Review of Quantitative Research and Software Applications in Assortment and Shelf Space Management." Omega40 (2): 199-209.

Inglay, R. S., and R. S. Dhalla. 2010. "Application of Systematic Layout Planning in Hypermarkets." Proceedings of the 2010 International Conference on Industrial Engineering and Operations Management, 9-10, Dhaka, Bangladesh. 
Kim, J.-G., and Y.-D. Kim. 2000. "Layout Planning for Facilities with Fixed Shapes and Input and Output Points.” International Journal of Production Research 38 (18): 4635-4653.

Komarudin, K., and Y. Wong. 2010a. "Applying Ant System for Solving Unequal Area Facility Layout Problems." European Journal of Operational Research 202 (3): 730-746.

Konak, A., S. Kulturel-Konak, B. A. Norman, and A. E. Smith. 2006. "A New Mixed Integer Programming Formulation for Facility Layout Design Using Flexible Bays.” Operations Research Letters 34 (6): 660-672.

Kuhn, H., and M. G. Sternbeck. 2013. "Integrative Retail Logistics: An Exploratory Study.” Operations Management Research 6 (1-2): $2-18$.

Kulturel-Konak, S. 2012. "A Linear Programming Embedded Probabilistic Tabu Search for the Unequal-Area Facility Layout Problem with Flexible Bays." European Journal of Operational Research223 (3): 614-625.

Lee, Y. H., and M. H. Lee. 2002. "A Shape-Based Block Layout Approach to Facility Layout Problems Using Hybrid Genetic Algorithm." Computers \& Industrial Engineering 42 (2-4): 237-248.

Liu, Q., and R. D. Meller. 2007. "A Sequence-Pair Representation and Mip-Model-Based Heuristic for the Facility Layout Problem with Rectangular Departments." IIE Transactions 39 (4): 377-394.

Meller, R. D., W. Chen, and H. D. Sherali. 2007. "Applying the Sequence-Pair Representation to Optimal Facility Layout Designs." Operations Research Letters 35 (5): 651-659.

Mohan, G., B. Sivakumaran, and P. Sharma. 2013. "Impact of Store Environment on Impulse Buying Behavior." European Journal of Marketing 47 (10): 1711-1732.

Montreuil, B. 1991. “A Modelling Framework for Integrating Layout Design and Flow Network Design.” In: White, J. A., Pence, I. W. (Eds.), Progress in Material Handling and Logistics. Vol. 2 of Material Handling. 95-115. Springer-Verlag.

Mou, S., D. J. Robb, and N. DeHoratius. 2017. "Retail Store Operations: Literature Review and Research Directions.” European Journal of Operational Research 265 (2): 399-422.

Mowrey, C. H., P. J. Parikh, and K. R. Gue. 2018. “A Model to Optimize Rack Layout in a Retail Store.” European Journal of Operational Research 271 (3): 1100-1112.

Nielsen. 2015. The Future of Grocery - E-Commerce, Digital Technology and Changing Shopping Preferences Around the World. Nielsen. https://www.nielsen.com/wp-content/uploads/sites/3/2019/04/nielsen-global-e-commerce-new-retail-report-april-2015.pdf.

Osman, H. M., M. E. Georgy, and M. E. Ibrahim. 2003. "A Hybrid Cad-Based Construction Site Layout Planning System Using Genetic Algorithms." Automation in Construction 12 (6): 749-764.

Ozcan, T., and S. Esnaf. 2013. “A Discrete Constrained Optimization Using Genetic Algorithms for a Bookstore Layout.” International Journal of Computational Intelligence Systems 6 (2): 261-278.

Ozgormus, E. 2015. "Optimization of Block Layout for Grocery Stores.” PhD thesis. Auburn, Alabama.

Paes, F. G., A. A. Pessoa, and T. Vidal. 2017. "A Hybrid Genetic Algorithm with Decomposition Phases for the Unequal Area Facility Layout Problem.” European Journal of Operational Research 256 (3): 742-756.

Palomo-Romero, J. M., L. Salas-Morera, and L. García-Hernández. 2017. “An Island Model Genetic Algorithm for Unequal Area Facility Layout Problems.” Expert Systems with Applications 68: 151-162.

Peng, C.-C. 2011. Optimizing the Layout of a Grocery/convenience Store to Maximize Revenue From Impulse Items. Buffalo: State University of New York.

Pinto, F., C. Soares, and P. Brazdil. 2015. "Combining Regression Models and Metaheuristics to Optimize Space Allocation in the Retail Industry.” Intelligent Data Analysis 19 (s1): S149-S162.

Pires, M., P. Amorim, J. Liz, and J. Pratas. 2015. "Design of Retail Backroom Storage: A Research Opportunity?” In Operations Research and Big Data, 167-174. Portugal: Springer.

Pires, M., P. Amorim, J. Liz, and J. Pratas. 2016. “A Framework for Designing Backroom Areas in Grocery Stores.” International Journal of Retail \& Distribution Management 45 (3): 230-252.

Pisinger, D., and M. Sigurd. 2007. "Using Decomposition Techniques and Constraint Programming for Solving the Two-Dimensional Bin-Packing Problem.” INFORMS Journal on Computing 19 (1): 36-51.

Scholz, D., A. Petrick, and W. Domschke. 2009. "Stats: a Slicing Tree and Tabu Search Based Heuristic for the Unequal Area Facility Layout Problem." European Journal of Operational Research 197 (1): 166-178.

Surjandari, I., and A. Seruni. 2010. "Design of Product Placement Layout in Retail Shop Using Market Basket Analysis." Makara Journal of Technology 9 (2): 43-47.

Tari, F. G., and H. Neghabi. 2015. "A New Linear Adjacency Approach for Facility Layout Problem with Unequal Area Departments." Journal of Manufacturing Systems 37: 93-103.

Turley, L. W., and J.-C. Chebat. 2002. "Linking Retail Strategy, Atmospheric Design and Shopping Behaviour." Journal of Marketing Management 18 (1-2): 125-144.

Van Zelst, S., K. Van Donselaar, T. van Woensel, R. Broekmeulen, and J. Fransoo. 2009. "Logistics Drivers for Shelf Stacking in Grocery Retail Stores: Potential for Efficiency Improvement.” International Journal of Production Economics 121 (2): 620-632.

Wang, M.-J., M. H. Hu, and M.-Y. Ku. 2005. "A Solution to the Unequal Area Facilities Layout Problem by Genetic Algorithm." Computers in Industry 56 (2): 207-220.

Wong, K. Y., and Komarudin. 2010b. "Solving Facility Layout Problems Using Flexible Bay Structure Representation and Ant System Algorithm.” Expert Systems with Applications 37 (7): 5523-5527. 
Wu, Y., and E. Appleton. 2002. "The Optimisation of Block Layout and Aisle Structure by a Genetic Algorithm.” Computers \& Industrial Engineering 41 (4): 371-387.

Yapicioglu, H., and A. E. Smith. 2012. “A Bi-Objective Model for the Retail Spatial Design Problem.” Engineering Optimization 44 (3): 243-266.

\section{Appendices \\ Appendix 1}

Sets and subsets
$K$
$K C$
$K T$
$F_{k}$
$D$

\section{Parameters}

$W(H)$

A

$w_{f k}\left(h_{f k}\right)$

$A_{f k}$

$r f_{k}$

$d_{d}^{x}\left(d_{d}^{y}\right)$

$f m_{d}$

$d s_{k d}^{x}\left(d s_{k d}^{y}\right)$

$s$

ma

$\Delta$

\section{Decision variables}

$\chi_{k f}$

$\gamma_{k d}$

$\alpha_{k i}\left(\delta_{k i}\right)$

$\psi_{k i}^{x}\left(\psi_{k i}^{y}\right)$

$a_{k}, b_{k}, c_{k}$ and $d_{k}$

$x_{k}\left(y_{k}\right)$

$\sigma_{k}^{x}\left(\sigma_{k}^{y}\right)$

$\xi_{k}^{x}\left(\xi_{k}^{y}\right)$

$v_{k d}^{x}\left(v_{k d}^{y}\right)$

$\omega_{k i}^{x}\left(\omega_{k i}^{y}\right)$ set of unequal area backroom departments to be placed subset of $K$ that only includes frozen departments subset of $K$ that only includes technical departments set of formats for each department set of doors

horizontal (vertical) dimensions of the floor space area of the floor space horizontal (vertical) dimensions of department $k$ in format $f$ area of department $k$ in format $f$

replenishment frequency of department $k$ position of door $d$ in horizontal (vertical) axis maximum flow of products allowed to go through door $d$ distance from door $d$ to department $k$ in the horizontal (vertical) axis minimum distance between departments minimum area in the centre of the backroom that should be left unoccupied maximum allowed difference in the departments' widths, or heights

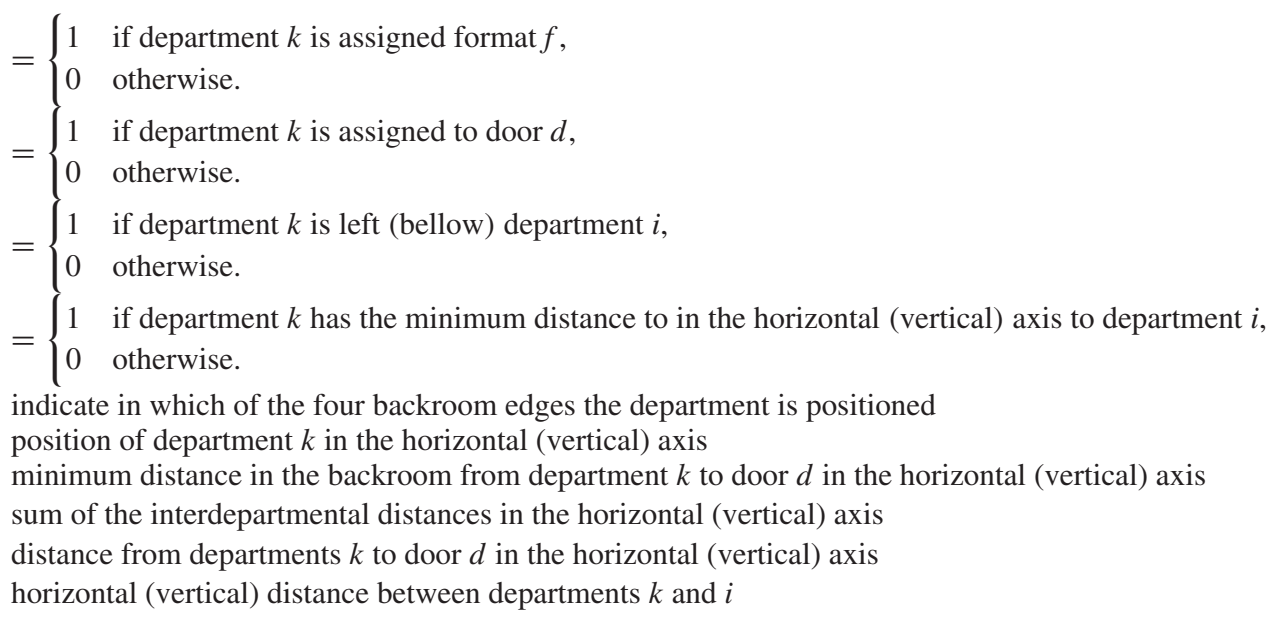

\section{Appendix 2}

\section{A.1. Master layout constraints}

Equations (2)-(4) defines the relative position of two departments in the backroom. More precisely, Equations (2) ensure that two departments can be left and/ or below another, Equations (3) ensure that if department $k$ is located left of department $i$ then the department $i$ can not be located left of department $k$. Similarly, in Equation (4) if department $k$ is located below of department $i$ then the department $i$ can not be located below of department $k$. In Figure A1 it is represented two departments positioned in the backroom and the respective coordinates. Department $k$ is located left of department $i$ and department $i$ is below $k$, which means that $\alpha_{k i}=1, \alpha_{i k}=0, \beta_{k i}=0$ and $\beta_{i k}=1$.

Constraints (5) are used to assign only one format for each department.

The guarantee that the departments do not overlap is ensured by Constraints (6) and (7). Constraints (6) stat that if department $k$ is on the left of department $i$, the $x$ coordinate of department $k\left(x_{k}\right)$ plus its width $\left(w_{f k}\right)$ has to be smaller than $x$ coordinate of department $i\left(x_{i}\right)$. 


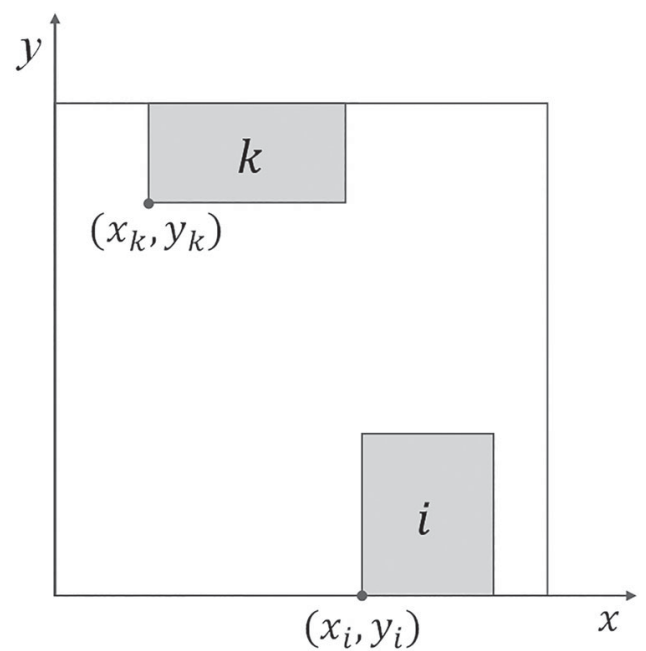

Figure A1. Example of two departments positioning.

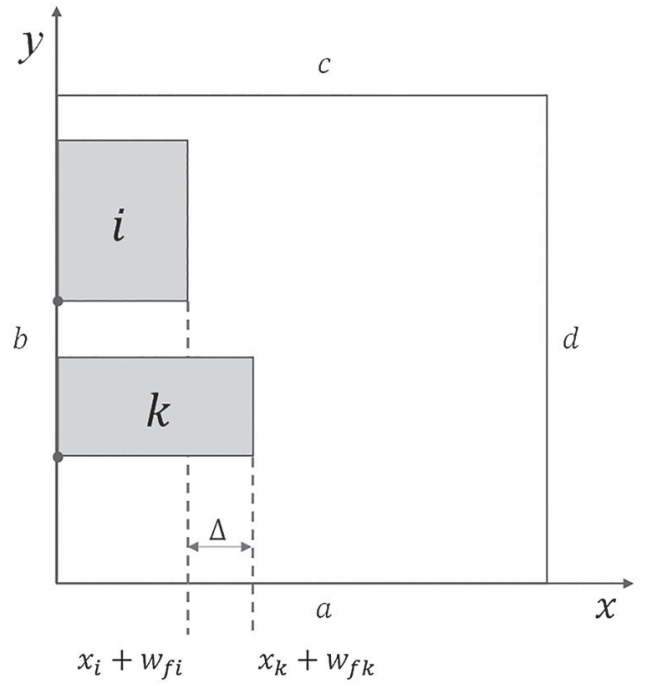

Figure A2. Alignment of the walls

If department $k$ is not on the left of department $i, x_{k}$ can take any value. In Figure A1 since $\alpha_{k i}=1, x_{k}$ plus $w_{f k}$ is smaller than $x_{i}$. In the same way, Constraints (7) state that if department $k$ is below department $i$, then the $y$ coordinate of department $k\left(y_{k}\right)$ plus its height $h_{f k}$, must be smaller than $\left(y_{i}\right)$.

Equations (8) and (9) ensure that each department $k$ is within the backroom space. Equation (10) ensures that the total area of formats selected for the departments does not exceed the available backroom space and that a minimum area is left for storing merchandise at ambient temperature.

It is intended that the departments that are in the same axis have the walls aligned, avoiding the construction of extra walls. Constraints (11)-(14) represent the alignment of the departments' walls. In Figure A2 are represented two departments $(i$ and $k)$ in the $y$-axis $\left(b_{i}=1, b_{k}=1\right)$, Constraint (11) ensures that the difference between the wall of department $k\left(x_{k}+w_{f k}\right)$ and department $i\left(x_{i}+w_{f i}\right)$ is smaller than a defined $\Delta$. Constraints (12)-(14) have the same purpose.

\section{A.2. Door-selection constraints}

The set of Constraints (15)-(20) are used to assign a door to each department, considering the total distance in $x$ and $y$-axis and a maximum products flow in each door. Constraints (15) are responsible for assigning a door to each department. In Constraints (16), the decision variable $v_{k d}^{x}$ gets the distance of department $k$ to door $d$ in the horizontal axis, the distance is calculated considering the middle of the department. Similarly, in Constraint (18) the decision variable $v_{k d}^{y}$ gets the vertical distance of department $k$ to door $d$, the distance is also 


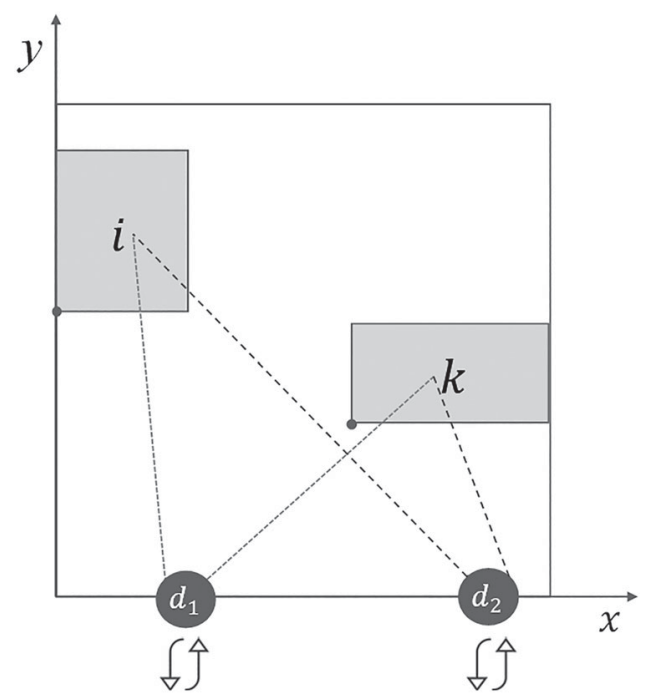

Figure A3. Door assignment.

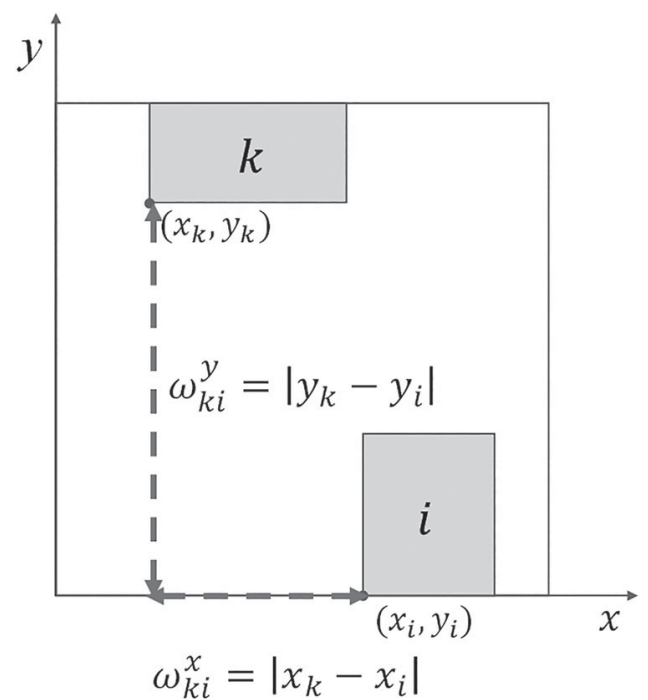

Figure A4. Distance between frozen departments.

calculated considering the middle of the department. The decisions variables $\sigma_{k}^{x}$ and $\sigma_{k}^{y}$ take the minimum distance in the backroom from department $k$ to door $d$ in the horizontal and vertical axis, considering Constraints (17) and (19) and its minimization in the objective function.

Constraints (20) ensure that the maximum products flow in each door is not exceeded (Figure A3).

\section{A.3. Energy constraints}

Constraints (21)-(24) are defined to consider energy efficiency aspects. To avoid heat loss, frozen departments should be located together. Decision variables $\omega_{k i}^{x}$ represent the horizontal distance between department $k$ and $i$ and decision variables $\omega_{k i}^{y}$ represent the vertical distance between department $k$ and $i$, both departments are of type frozen. Constraints (21) and (23) are used to obtain these values. In Figure A4, it is presented an example with two departments and its horizontal and vertical distances.

Decision variables $\xi_{k}^{x}$ and $\xi_{k}^{y}$ represent the total horizontal and vertical distance between department $k$ and all the other departments (Constraints (22) and (24)) and are minimised in the objective function. 


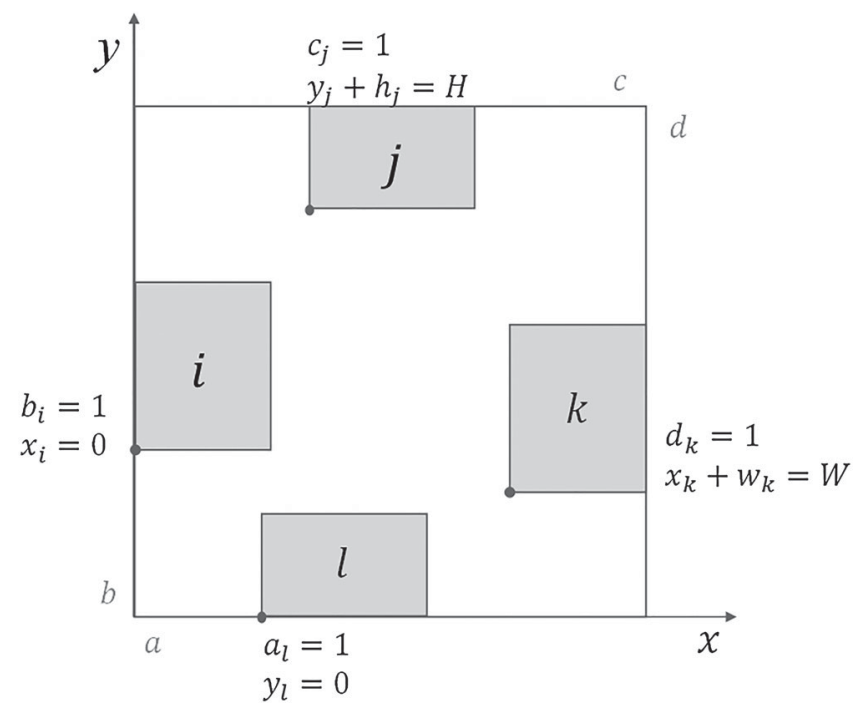

Figure A5. Positioning of departments in the backroom perimeter.

\section{A.4. Backroom perimeter constraints}

Constraints $(25)^{2}-(29)$ ensure that the departments are located in the perimeter of the backroom. Constraint (25) ensure that the department should be located in at least one edge of the backroom. If the department is located in the $a$ edge of the backroom the $y$ coordinate is fixed to zero, this is ensured by Constraint (26) in Figure A5, $l$ department is located in edge $a\left(a_{l}=1\right)$, which means that $y_{l}=0$. If the department is located in the $b$ edge of the backroom, $x$ coordinate is equal to zero, this is guaranteed by Constraint (27). In Figure A5 the department $i$ is located in the $b$ edge $\left(b_{i}=1\right)$ and $x_{i}=0$. Whenever a department is located in the $c$ edge of the backroom, the decision variable $c$ is equal to one, and the $y$ coordinate of the department plus its height are equal to the height of the backroom. An example can be found in the department $j$ in Figure A5. Similarly, if a department is located in the $d$ edge of the backroom, decision variable $d_{k}$ is equal to one, and the sum of the $x$ position of the department with its width is equal to the width of the backroom. In Figure A5, an example of this constraint could be found considering the location of department $k$.

\section{A.5. Store operations constraints}

It is required that the area that links all the departments is a single undivided polygon with enough space to move the pallet truck, facilitating the store operations. Since the departments are located in the edges of the backroom, it should be ensured that there is a minimum distance between departments in different edges. Considering the backroom rectangle $a, b, c, d$, the minimum distance should be ensured in six pairs $(a b, b c, c d, a d, a c, b d)$. The constraints for each pair of edges are presented below.

\section{A.5.1. Pair $a b$}

Constraints (A1) ensures that if department $i$ is in edge $a\left(a_{i}=1\right)$ and department $k$ is in edge $b\left(b_{k}=1\right)$, a minimum distance should exist from department $k$ to $i$ in the vertical and/or horizontal axis. If a minimum horizontal distance should exist between department $i$ and $k$ if $a_{i}=1$ and $b_{k}=1$, Constraint (A2) ensure that the $x$ coordinate of department $i$ has to be greater than the end of department $k$ plus a minimum distance $s$. If a minimum vertical distance is required between department $i$ and $k$ if $a_{i}=1$ and $b_{k}=1$, Constraint (A3) ensure that the $y$ coordinate for department $k$ should be greater than the end $y$ coordinate of department $i$ plus the minimum distance $s$.

An example with the location of departments $i$ and $k$ in the edges $a$ and $b$ is presented in Figure A6.

$$
\begin{aligned}
\psi_{k i}^{x}+\psi_{k i}^{y} & \geqslant a_{i}+b_{k}-1, \quad \forall i, k \in K: k \neq i \\
x_{k} & \leqslant x_{i}-\sum_{f} \chi_{k f} \cdot w_{f k}-s+M\left(1-\psi_{k i}^{x}\right), \quad \forall i, k \in K: k \neq i \\
y_{k} & \geqslant y_{i}+\sum_{f} \chi_{i f} \cdot h_{f i}+s-M\left(1-\psi_{k i}^{y}\right), \quad \forall i, k \in K: k \neq i
\end{aligned}
$$

\section{A.5.2. Pair $b c$}

Constraints for the pair of edges $b c$ are similar to the ones for pair $a b$, considering the relative positions of departments $i$ and $k$ in the edge $b$ and $c$, respectively.

$$
x_{k} \geqslant x_{i}+\sum_{f} \chi_{i f} \cdot w_{f i}+s-M\left(1-\psi_{k i}^{x}\right), \quad \forall i, k \in K: k \neq i
$$



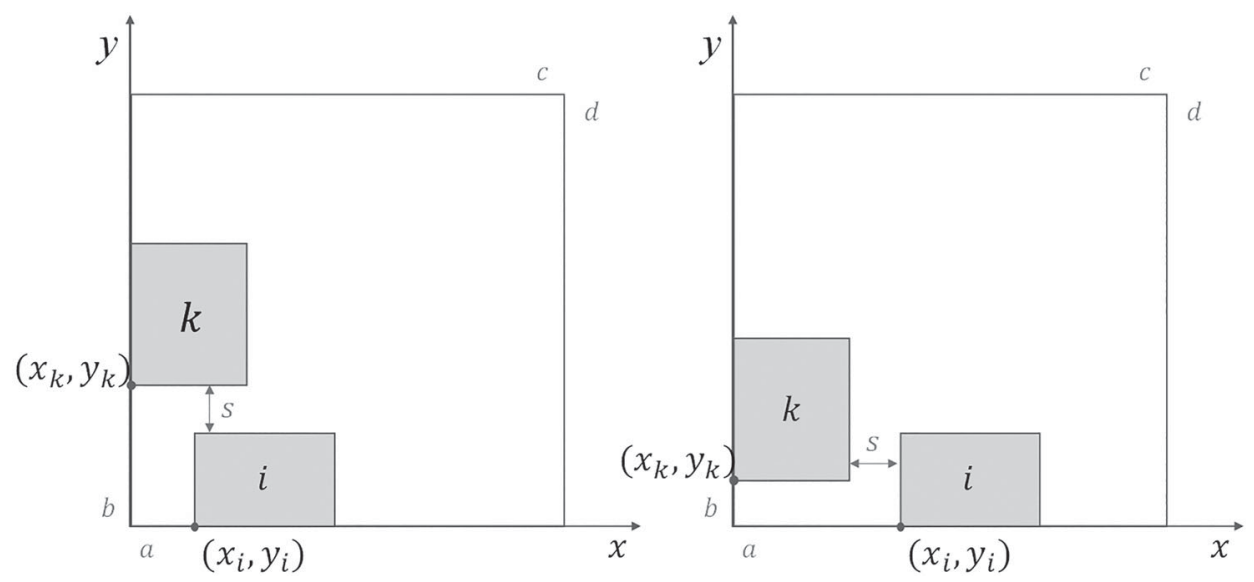

Figure A6. Minimum distance for departments in edge $a$ and $b$.

$$
\begin{aligned}
y_{k} & \geqslant y_{i}+\sum_{f} \chi_{i f} \cdot h_{f i}+s-M\left(1-\psi_{k i}^{y}\right), \quad \forall i, k \in K: k \neq i \\
\psi_{k i}^{x}+\psi_{k i}^{y} & \geqslant b_{i}+c_{k}-1, \quad \forall i, k \in K: k \neq i
\end{aligned}
$$

\section{A.5.3. Pair cd}

Constraints for the pair of edges $c d$ are similar to the ones for pair $a b$, considering the relative positions of departments $i$ and $k$ in the edge $c$ and $d$, respectively.

$$
\begin{aligned}
x_{k} & \geqslant x_{i}+\sum_{f} \chi_{i f} \cdot w_{f i}+s-M\left(1-\psi_{k i}^{x}\right), \quad \forall i, k \in K: k \neq i \\
y_{k} & \leqslant y_{i}-\sum_{f} \chi_{k f} \cdot h_{f k}-s+M\left(1-\psi_{k i}^{y}\right), \quad \forall i, k \in K: k \neq i \\
\psi_{k i}^{x}+\psi_{k i}^{y} & \geqslant c_{i}+d_{k}-1, \quad \forall i, k \in K: k \neq i
\end{aligned}
$$

\section{A.5.4. Pair ad}

Constraints for the pair of edges $a d$ are similar to the ones for pair $a b$, considering the relative positions of departments $i$ and $k$ in the edge $a$ and $d$, respectively.

$$
\begin{aligned}
x_{k} & \geqslant x_{i}+\sum_{f} \chi_{i f} \cdot w_{f i}+s-M\left(1-\psi_{k i}^{x}\right), \quad \forall i, k \in K: k \neq i \\
y_{k} & \geqslant y_{i}+\sum_{f} \chi_{i f} \cdot h_{f i}+s-M\left(1-\psi_{k i}^{y}\right), \quad \forall i, k \in K: k \neq i \\
\psi_{k i}^{x}+\psi_{k i}^{y} & \geqslant a_{i}+d_{k}-1, \quad \forall i, k \in K: k \neq i
\end{aligned}
$$

\section{A.5.5. Pair ac}

Constraints for the pair of edges $a c$ are similar to the ones for pair $a b$, considering the relative positions of departments $i$ and $k$ in the edge $a$ and $c$, respectively.

$$
\begin{aligned}
& y_{k} \geqslant y_{i}+\sum_{f} \chi_{i f} \cdot h_{f i}+s-M\left(1-\psi_{k i}^{y}\right), \quad \forall i, k \in K: k \neq i \\
& x_{k} \leqslant x_{i}-\sum_{f} \chi_{k f} \cdot w_{f k}-s+M\left(1-\psi_{k i}^{x}\right), \quad \forall i, k \in K: k \neq i
\end{aligned}
$$




$$
\begin{gathered}
x_{k} \geqslant x_{i}+\sum_{f} \chi_{i f} \cdot w_{f i}+s-M\left(1-\psi_{k i}^{\prime x}\right), \quad \forall i, k \in K: k \neq i \\
\psi_{k i}^{x}+\psi_{k i}^{\prime x}+\psi_{k i}^{y} \geqslant a_{i}+c_{k}-1, \quad \forall i, k \in K: k \neq i
\end{gathered}
$$

\section{A.5.6. Pair bd}

Constraints for the pair of edges $b d$ are similar to the ones for pair $a b$, considering the relative positions of departments $i$ and $k$ in the edge $b$ and $d$, respectively.

$$
\begin{gathered}
x_{k} \geqslant x_{i}+\sum_{f} \chi_{i f} \cdot w_{f i}+s-M\left(1-\psi_{k i}^{x}\right), \quad \forall i, k \in K: k \neq i \\
y_{k} \geqslant y_{i}+\sum_{f} \chi_{i f} \cdot h_{f i}+s-M\left(1-\psi_{k i}^{y}\right), \quad \forall i, k \in K: k \neq i \\
y_{k} \leqslant y_{i}-\sum_{f} \chi_{k f} \cdot h_{f k}-s+M\left(1-\psi_{k i}^{y}\right), \quad \forall i, k \in K: k \neq i \\
\psi_{k i}^{x}+\psi_{k i}^{y}+\psi_{k i}^{\prime} \geqslant b_{i}+d_{k}-1, \quad \forall i, k \in K: k \neq i
\end{gathered}
$$

Analysing Constraints (A1)-(A20) it can be observed that some constraints are repeated. Summing up, the set of constraints required to ensure store operations are presented below.

$$
\begin{gathered}
x_{k} \leqslant x_{i}-\sum_{f} \chi_{k f} \cdot w_{f k}-s+M\left(1-\psi_{k i}^{x}\right), \quad \forall i, k \in K: k \neq i \\
x_{k} \geqslant x_{i}+\sum_{f} \chi_{i f} \cdot w_{f i}+s-M\left(1-\psi_{k i}^{x}\right), \quad \forall i, k \in K: k \neq i \\
y_{k} \geqslant y_{i}+\sum_{f} \chi_{i f} \cdot h_{f i}+s-M\left(1-\psi_{k i}^{y}\right), \quad \forall i, k \in K: k \neq i \\
y_{k} \leqslant y_{i}-\sum_{f} \chi_{k f} \cdot h_{f k}-s+M\left(1-\psi_{k i}^{y}\right), \quad \forall i, k \in K: k \neq i \\
\psi_{k i}^{x}+\psi_{k i}^{y} \geqslant a_{i}+b_{k}-1, \quad \forall i, k \in K: k \neq i \\
\psi_{k i}^{x}+\psi_{k i}^{y} \geqslant b_{i}+c_{k}-1, \quad \forall i, k \in K: k \neq i \\
\psi_{k i}^{x}+\psi_{k i}^{y} \geqslant c_{i}+d_{k}-1, \quad \forall i, k \in K: k \neq i \\
\psi_{k i}^{x}+\psi_{k i}^{y} \geqslant a_{i}+d_{k}-1, \quad \forall i, k \in K: k \neq i \\
\psi_{k i}^{x}+\psi_{k i}^{\prime x}+\psi_{k i}^{y} \geqslant a_{i}+c_{k}-1, \quad \forall i, k \in K: k \neq i \\
\psi_{k i}^{x}+\psi_{k i}^{y}+\psi_{k i}^{y} \geqslant b_{i}+d_{k}-1, \quad \forall i, k \in K: k \neq i
\end{gathered}
$$

\section{Appendix 3}

The models used to develop each of the scenarios described in Section 5.3 is presented in this appendix.

To develop the first scenario (S1), which does not consider the distance to the sales area, the objective function needs to be adjusted. Thus, the parcel concerning the location of the departments in the sales area is removed from the objective function (please refer to Equation (A31)). To develop the second scenario (S2), which does not consider the energy efficiency issues, the parcel concerning the distance between the frozen departments is removed from the objective function (S2 in Equation (A31)). Scenario 3 (S3), which ignores the different replenishment frequencies of departments, is obtained by removing $r f_{k}$ from the objective function A.

$$
\text { Minimize } \sum_{k \in K \backslash\{K T\}}(\sigma_{k}^{x}+\sigma_{k}^{y}+\sum_{d \in D} \underbrace{\left.\left(d s_{k d}^{x}+d s_{k d}^{y}\right) \cdot \gamma_{k d}\right) \cdot \overbrace{r f_{k}}^{\mathrm{S} 3}}_{\mathrm{S} 1}+\overbrace{\sum_{k \in K C}\left(\xi_{k}^{x}+\xi_{k}^{y}\right)}^{\mathrm{S} 2}
$$




\section{Appendix 4}

Table A1. Characterization of store G.

\begin{tabular}{llrrr}
\hline \# Dept & \multicolumn{1}{c}{ Function } & Size $\left(\mathrm{m}^{2}\right)$ & R.F. & \# Formats \\
\hline 1 & Store dairy & 21 & 1 & 2 \\
2 & Store delicatessen & 28 & 2 & 4 \\
3 & Store fruits and vegetables & 15 & 19 & 3 \\
4 & Store fish & 17 & 16 & 2 \\
5 & Store bakery & 20 & 2 & 4 \\
6 & Store meat & 20 & 18 & 4 \\
7 & Store poultry & 12 & 5 & 2 \\
8 & Store codfish & 10 & 4 & 2 \\
9 & Store frozen food & 30 & 2 & 4 \\
10 & Store frozen bakery & 21 & 2 & 2 \\
11 & Prepare meat & 19 & 25 & 2 \\
12 & Prepare take-away & 17 & 25 & 2 \\
13 & Prepare bakery & 18 & 25 & 2 \\
14 & Washing area & 15 & - & 3 \\
15 & Machine room 1 & 50 & - & 5 \\
16 & Machine room 2 & 15 & - & 3 \\
17 & Machine room 3 & 18 & - & 2 \\
18 & Decoration room & 25 & - & 1 \\
19 & Meting room & 30 & - & 4 \\
20 & Store manager office & 20 & - & 4 \\
21 & Department managers office & 40 & - & 6 \\
22 & Lunchroom & 33 & - & 5 \\
23 & Male dressing room & 40 & - & 6 \\
24 & Feminine dressing room & 50 & - & 5 \\
25 & Archive & 12 & - & 2 \\
26 & Pharmacy warehouse & 8 & - & 2 \\
\hline
\end{tabular}

Caption: R.F. - Replenishment frequencies. 